\title{
Frank Aggregation Operators for Triangular Interval Type-2 Fuzzy Set and Its Application in Multiple Attribute Group Decision Making
}

\author{
Jindong Qin and Xinwang Liu \\ School of Economics and Management, Southeast University, Nanjing, Jiangsu 211189, China \\ Correspondence should be addressed to Xinwang Liu; xwliu@seu.edu.cn
}

Received 18 February 2014; Accepted 28 June 2014; Published 2 September 2014

Academic Editor: Hung-Yuan Chung

Copyright (c) 2014 J. Qin and X. Liu. This is an open access article distributed under the Creative Commons Attribution License, which permits unrestricted use, distribution, and reproduction in any medium, provided the original work is properly cited.

\begin{abstract}
This paper investigates an approach to multiple attribute group decision-making (MAGDM) problems, in which the individual assessments are in the form of triangle interval type-2 fuzzy numbers (TIT2FNs). Firstly, some Frank operation laws of triangle interval type-2 fuzzy set (TIT2FS) are defined. Secondly, some Frank aggregation operators such as the triangle interval type-2 fuzzy Frank weighted averaging (TIT2FFWA) operator and the triangle interval type-2 fuzzy Frank weighted geometric (TIT2FFWG) operator are developed for aggregation TIT2FNs. Furthermore, some desirable properties of the two aggregation operators are analyzed in detail. Finally, an approach based on TIT2FFWA (or TIT2FFWG) operator to solve MAGDM is developed. An illustrative example about supplier selection is provided to illustrate the developed procedures. The results demonstrate the practicality and effectiveness of our new method.
\end{abstract}

\section{Introduction}

Fuzzy multiple attribute group decision making (FMAGDM) is a hot area of research in fuzzy decision analysis theory. It involves the process to select the best alternative(s) from the set of feasible alternatives with respect to multiple attributes, in which the decision information usually with fuzziness or uncertainty is provided by a group of decision makes under fuzzy environment. In recent years, many techniques have been developed to solve FMAGDM problems [1-8]. Li and Yang [1] developed a linear programming technique for multidimensional analysis of preferences in multiple attribute group decision making under fuzzy environments. Wang and Lee [2] generalized TOPSIS method for FMAGDM based on high and low operators which satisfy the partial ordering relation on fuzzy numbers. Xu and Chen [3] established an interactive model for FMAGDM in which the information about attribute weights is partly known. Lin and $\mathrm{Wu}$ [4] presented a fuzzy DEMATEL method for group decision making to gather group ideas and analyzed the cause-effect relationship of complex problems in fuzzy environment. Guha and Chakraborty [5] developed a FMAGDM technique considering the degrees of confidence of experts' opinions and aggregated the decision information by using similarity. Hatami-Marbini and Tavana [6] proposed an alternative fuzzy outranking method by extending the ELECTRE I method to take into account the uncertain, imprecise, and linguistic assessments provided by a group of decision makers. Mousavi et al. [7] utilized Monte Carlo simulation technique to derive a stochastic approach for handling FMAGDM and applied it in risk selection problem in a highway project. Zhao et al. [8] utilized the fuzzy prioritized operators to develop some models for triangular FMAGDM in which both the attributes and decision makes are in different priority level.

Type-2 fuzzy set (T2FS) was first proposed by Zadeh [9], which can be viewed as an effective extension of tradition type-1 fuzzy set (T1FS). The membership function of T2FS, which is characterized by primary function, secondary function and a footprint of uncertainty (FOU). It is the new third dimension of T2FS and the footprint of uncertainty can reflect more additional degrees of freedom that make it more capable of handling imprecision and imperfect information in real-world application [10-12]. Hence, T2FS are receiving more and more attentions from researchers and have been 
successfully developed both in theoretical and in practical aspects [13-21]. However, because the computational complexity of general T2FS is too high, it is usually difficult to use in real practice situations [22]. To overcome the limitation of T2FS, interval type-2 fuzzy set (IT2FS), which is also called interval-valued fuzzy set (IVFS) and contains membership values that are crisp interval from zero to one, is proposed [23] and has been the most useful tool for modeling uncertainty and complexity in many domains [2430]. In particular, many approaches have been presented in the field of multiple attribute group decision making (MAGDM). Chen and Lee [31] presented a new method based on arithmetic operations for handing multiple attribute hierarchical group decision-making problems. Chen and Lee [32] further developed an interval type-2 TOPSIS method to solve MAGDM and gave some practical examples to illustrate the procedure of the proposed method. Chen et al. [33] presented a ranking formula for IT2FS information and established a framework based on ranking method for handling MAGDM with interval type-2 fuzzy information. Hu et al. [34] presented an approach based on possibility degree for interval type-2 fuzzy multiple criteria group decision making and established a maximizing deviation optimal model for determining the attribute weights, in which the weight information is partly known. Wang et al. [35] established some weight optimal models to determine the weights of attributes and utilized the interval type-2 weighted averaging (IT2WA) operator for handling MAGDM under interval type-2 fuzzy environment. Chen [36] presented a signed-distance-based method for determining the objective importance of criteria and handling fuzzy multiple criteria group decision-making problems in a flexible and intelligent way. Chen et al. [37] investigated extended QUALIFLEX method for group decision making, in which the attribute values take the form of IT2FS, and further applied it in medical decision making. Baležentis and Zeng [38] further extended MULTIMOORA method with IT2FS and applied it in the field of human resource management and performance management. Chiao [39] introduced the concepts of triangular interval type2 fuzzy set and developed the analytic hierarchy process (AHP) method based on logarithmic regression function for handling MAGDM problem. Ngan [40] established the probabilistic linguistic framework with IT2FS MAGDM problems, and some arithmetic operations, such as union and intersection between the interval type- 2 fuzzy linguistic numbers, were also defined. Chen [41] developed a new linear assignment method based on signed distances for handling MAGDM problems with IT2FS information and applied it in the selection of landfill site. Z. M. Zhang and S. H. Zhang [42] proposed the concept of trapezoidal interval type-2 fuzzy soft set and further developed an MAGDM approach under interval type-2 fuzzy environment.

For MAGDM problems, an essential important step is how to aggregate the decision-making information in different formats with aggregation operators. As an important tool for information fusion, aggregation operators are widely used in various fuzzy set (FS), such as T1FS, intuitionistic fuzzy set (IFS), and hesitant fuzzy set (HFS). However, up till now, the researches on type-2 fuzzy aggregation operators are very rare. Wu and Mendel [43] presented the concept of linguistic weighted average (LWA), in which the attributes and the weights both are in the form of IT2FS, the algorithm was provided and verified the LWA operator can be decomposed into finding two fuzzy weighted average (FWA). Liu et al. [44] presented an analytical solution method for the FWA; the method had some good mathematical analysis properties, which can be used for interval type- 2 aggregation process. Zhou et al. $[45,46]$ proposed the type-2 OWA operator to aggregate the linguistic opinions or preferences in human decision-making modeling by T2FS, and a direct method to aggregate IT2FS by type-2 OWA operator was provided. Liu et al. [47-49] developed some aggregation operators, such as weighted aggregation operators, geometric aggregation operators, and hybrid harmonic averaging operators to handle MAGDM problems with interval-valued trapezoidal fuzzy numbers.

All the above interval type-2 fuzzy aggregation operators are based on alpha-cut decomposition theorem [9]; the main difference between the existing interval type-2 fuzzy aggregation operators and other FS aggregation operators is that the interval type-2 fuzzy aggregation operators are derived by fuzzy extension principle while the other fuzzy aggregation operators are obtained by triangle norms $[50,51]$. As the fundamental of information fusion, triangle norms play an important role and have been successfully used to derive various fuzzy aggregation operators [52-54]. However, until now, there is no research about interval type-2 aggregation operator based on triangle norm operations. In order to fill this gap, we will utilize the Frank triangle norms to develop some desirable interval type-2 fuzzy aggregation operators in this paper.

Frank triangle norms [55], which is the only one type triangle norm satisfying the compatibility. Since the Frank triangle norms involve the parameter, this can provide more flexibility and robustness in the process of information fusion and make it more adequate to model practical decisionmaking problems than other triangle norms [56]. Calvo et al. [57] study the functional equations of Frank and Alsina for two classes of commutative, associative, and increasing binary operators. Yager [58] introduced the extensions operations to the lattice of closed interval-valued fuzzy set based on Frank $t$-norms and gave necessary and sufficient conditions such that these operations can form a complete algebraic structure. Casanovas and Torrens [59] investigated the additive generating function of Frank $t$-norms and established a framework with Frank $t$-norms in approximate reasoning. Sarkoci [60] made a comparison between the Frank $t$-norms and the Hamacher $t$-norms and proved that two different $t$ norms form the same family. However, the previous research works almost focus on the basic mathematical construct and properties of Frank $t$-norms; the researches of its applications are very rare, especially in aggregation and decision making. To date, we have not seen any researches on aggregation operators based on Frank $t$-norms for decision-making problems. Therefore, it is meaningful to study aggregation operators based on Frank triangle norms operations and their application in MAGDM under triangle interval type-2 fuzzy environment. 
The remainder of this paper is shown as follows. In Section 2, we briefly introduce some basic concepts of T2FS, triangle interval type-2 fuzzy set, and Frank triangle norms. In Section 3, we establish the Frank operational laws of triangle interval type-2 fuzzy set. In Section 4, we present triangle interval type-2 fuzzy Frank weighted averaging (TIT2FFWA) operator and triangle interval type-2 fuzzy Frank geometric average (TIT2FFWG) operator and also discuss some desirable properties in detail. In Section 5, we develop an approach based on the proposed Frank aggregation operators to MAGDM under the triangle interval type-2 fuzzy environment. A practical example is provided to illustrate the procedure of our method in Section 6. Finally, we end the paper with some conclusions and point out future researches in Section 7.

\section{Preliminaries}

In this section, we will briefly review some basic concepts of type-2 fuzzy set and Frank triangular norms.

\subsection{Type-2 Fuzzy Set}

Definition 1 (see [23]). A type-2 fuzzy set $\widetilde{A}$ in the universe of discourse $X$ can be represented by a type- 2 membership function $\mu_{A}(x, u)$ as follows:

$$
\widetilde{A}=\left\{\left((x, u), \mu_{A}(x, u)\right) \mid \forall x \in X, \forall u \in J_{x} \subseteq[0,1]\right\},
$$

where $J_{x}$ denotes an interval in $[0,1]$. Moreover, the type-2 fuzzy set can also be expressed as the following form:

$$
\widetilde{A}=\int_{x \in X} \int_{u \in J_{x}} \frac{\mu_{A}(x, u)}{(x, u)}=\int_{x \in X} \frac{\left(\int_{u \in J_{x}} \mu_{A}(x, u) / u\right)}{x},
$$

where $J_{x} \subseteq[0,1]$ is the primary membership at $x$, and $\int_{u \in J_{x}} \mu_{A}(x, u) / u$ indicates the second membership at $x$. For discreet situations, $\int$ is replaced by $\sum$.

Definition 2 (see [23]). Let $\widetilde{A}$ be a type-2 fuzzy set in the universe of discourse $X$ represented by a type- 2 membership function $\mu_{A}(x, u)$. If all $\mu_{A}(x, u)=1$, then $\widetilde{A}$ is called an interval type-2 fuzzy set (IT2FS). An interval type-2 fuzzy set can be regarded as a special case of the type- 2 fuzzy set, which is defined as

$$
\widetilde{A}=\int_{x \in X} \int_{u \in J_{x}} \frac{1}{(x, u)}=\int_{x \in X} \frac{\left(\int_{u \in J_{x}} 1 / u\right)}{x} .
$$

It is obvious that the interval type-2 fuzzy set $\widetilde{A}$ defined on $X$ is completely determined by the primary membership which is called the footprint of uncertainty (FOU), and the FOU can be expressed as follows:

$$
\operatorname{FOU}(\widetilde{A})=\bigcup_{x \in X} J_{x}=\bigcup_{x \in X}\left\{(x, u) \mid u \in J_{x} \subseteq[0,1]\right\} .
$$

2.2. Triangular Interval Type-2 Fuzzy Set. Because the operations on interval type-2 fuzzy sets are very complex according to the decomposition theorem [23], the interval type-2 fuzzy sets are usually taken in some simplified formations in applications. Here, we follow the results of Chiao [39], which adopted triangular interval type-2 fuzzy set for solving MAGDM problems.

In [39], Chiao proposed the concept of triangular interval type-2 fuzzy set, in which the upper membership function (UMF) and the lower membership function (LMF) are represented by triangular fuzzy number.

Let $\widetilde{A}=\left(\left[\underline{l_{A}}, \overline{l_{A}}\right], m_{A},\left[\underline{r_{A}}, \overline{r_{A}}\right]\right)$ be a triangular interval type-2 fuzzy set defined on $\bar{X}$, which is shown in Figure 1, where $\underline{l_{A}}, \overline{l_{A}}, m_{A}, \underline{r_{A}}, \overline{r_{A}}$ are the reference points of the triangular interval type-2 fuzzy set, satisfying $0 \leq l_{A} \leq \overline{l_{A}} \leq m_{A} \leq$ $r_{A} \leq \overline{r_{A}} \leq 1$. The upper and lower membership functions of $\overline{\widetilde{A}}$ are defined as

$$
\begin{aligned}
& \mathrm{UMF}_{\widetilde{A}}(x)= \begin{cases}\frac{x-\underline{l_{A}}}{m_{A}-\underline{l_{A}}} & \underline{l_{A}} \leq x<m_{A}, \\
1 & x=m_{A}, \\
\frac{x-\overline{r_{A}}}{m_{A}-\overline{r_{A}}} & m_{A} \leq x<\overline{r_{A}},\end{cases} \\
& \operatorname{LMF}_{\widetilde{A}}(x)= \begin{cases}\frac{x-\overline{l_{A}}}{m_{A}-\overline{l_{A}}} & \overline{l_{A}} \leq x<m_{A}, \\
1 & x=m_{A}, \\
\frac{x-\underline{r_{A}}}{m_{A}-\underline{r_{A}}} & m_{A} \leq x<\underline{r_{A}} .\end{cases}
\end{aligned}
$$

The footprint of uncertainty (FOU) of the membership function of $\widetilde{A}$ is shown as the shaded region in Figure 1. Obviously, if $\underline{l_{A}}=\overline{l_{A}}, \underline{r_{A}}=\overline{r_{A}}$, then $\operatorname{UMF}_{\widetilde{A}}(x)=\operatorname{LMF}_{\widetilde{A}}(x)$ for all $x \in X$, and the triangular interval type-2 fuzzy set reduces to the triangular type-1 fuzzy set.

If $X$ is a set in which the elements are all real numbers, then a triangular interval type-2 fuzzy set in $X$ is called a triangular interval type-2 fuzzy number (TIT2FN).

Chen et al. [33] proposed the ranking formula for interval type-2 fuzzy number as follows.

Definition 3 (see [33]). Let $\widetilde{A}=\left(\left[l_{A}, \overline{l_{A}}\right], m_{A},\left[r_{A}, \overline{r_{A}}\right]\right)$ be a triangular interval type-2 fuzzy number in Figure 1; the ranking value $\operatorname{Rank}(\widetilde{A})$ of the triangular interval type- 2 fuzzy number $\widetilde{A}$ is defined as follows:

$$
\operatorname{Rank}(\widetilde{A})=\left(\frac{\underline{\underline{l_{A}}+\overline{r_{A}}}}{2}+1\right) \times \frac{\underline{\underline{l_{A}}+\overline{l_{A}}+\underline{r_{A}}+\overline{r_{A}}+4 m_{A}}}{8} .
$$

The larger the ranking value $\operatorname{Rank}(\widetilde{A})$, the greater the TIT2FN $\widetilde{A}$.

2.3. Frank Triangular Norms. Frank operations include the Frank product and Frank sum, which are examples of triangle 


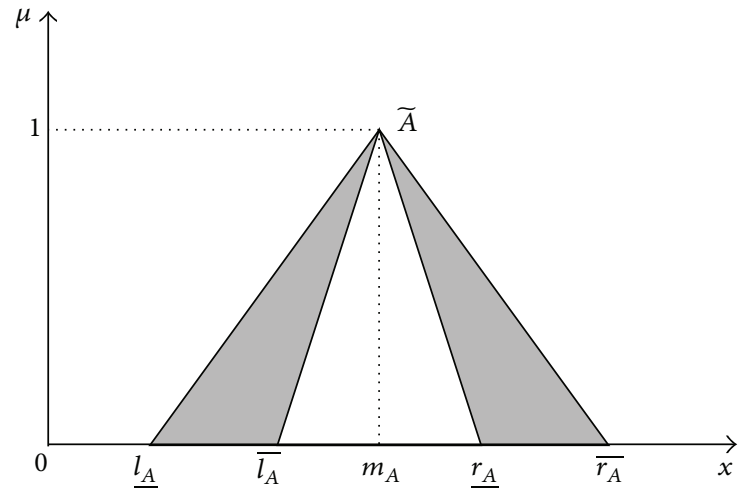

Figure 1: A triangular interval type-2 fuzzy set.

norms and triangle conorms, respectively. Frank product $\bigotimes_{F}$ is a $t$-norm and Frank sum $\bigoplus_{F}$ is a $t$-conorm, and the mathematical forms are defined as follows [55]:

$$
\begin{gathered}
a \bigoplus_{F} b=1-\log _{\lambda}\left(1+\frac{\left(\lambda^{1-a}-1\right)\left(\lambda^{1-b}-1\right)}{\lambda-1}\right) \lambda>1, \\
\forall(a, b) \in[0,1]^{2} \\
a \bigotimes_{F} b=\log _{\lambda}\left(1+\frac{\left(\lambda^{a}-1\right)\left(\lambda^{b}-1\right)}{\lambda-1}\right) \lambda>1, \\
\forall(a, b) \in[0,1]^{2} .
\end{gathered}
$$

It is pointed out that the Frank product and Frank sum have the following property [61]:

$$
\begin{aligned}
& \left(a \bigoplus_{F} b\right)+\left(a \bigotimes_{F} b\right)=a+b \\
& \frac{\partial\left(a \bigoplus_{F} b\right)}{\partial a}+\frac{\partial\left(a \bigotimes_{F} b\right)}{\partial a}=1
\end{aligned}
$$

Based on the limit theory, we can easily prove the interesting results as follows [61].

(1) If $\lambda \rightarrow 1$, then $a \bigoplus_{F} b \rightarrow a+b-a b, a \bigotimes_{F} b \rightarrow a b$, and the Frank product and Frank sum are reduced to the probability product and probability sum.

(2) If $\lambda \rightarrow \infty$, then $a \bigoplus_{F} b \rightarrow \min (a+b, 1), a \bigotimes_{F} b \rightarrow$ $\max (0, a+b-1)$, and the Frank product and Frank sum are reduced to the Lukasiewicz product and Lukasiewicz sum.

\section{Frank Operations of Triangular Interval Type-2 Fuzzy Numbers}

Based on Frank $t$-corm and Frank $t$-conorm previously mentioned, we will establish the basic operation laws of triangular interval type-2 fuzzy numbers in this section.
Definition 4. Let $\widetilde{A}, \widetilde{A}_{1}$, and $\widetilde{A}_{2}$ be three TIT2FNs, and $\lambda>1$; then we define their operation laws as follows.

(1) Addition operation:

$$
\begin{aligned}
& \widetilde{A}_{1} \bigoplus_{F} \widetilde{A}_{2} \\
& =\left(\left[1-\log _{\lambda}\left(1+\frac{\prod_{j=1}^{2}\left(\lambda^{1-l_{A_{j}}}-1\right)}{\lambda-1}\right),\right.\right. \\
& \left.1-\log _{\lambda}\left(1+\frac{\prod_{j=1}^{2}\left(\lambda^{1-\overline{l_{A_{j}}}}-1\right)}{\lambda-1}\right)\right], \\
& 1-\log _{\lambda}\left(1+\frac{\prod_{j=1}^{2}\left(\lambda^{1-m_{A_{j}}}-1\right)}{\lambda-1}\right), \\
& {\left[1-\log _{\lambda}\left(1+\frac{\prod_{j=1}^{2}\left(\lambda^{1-r_{A_{j}}}-1\right)}{\lambda-1}\right),\right.} \\
& \left.\left.1-\log _{\lambda}\left(1+\frac{\prod_{j=1}^{2}\left(\lambda^{1-\overline{r_{A_{j}}}}-1\right)}{\lambda-1}\right)\right]\right) .
\end{aligned}
$$

(2) Multiplication operation:

$$
\begin{aligned}
\widetilde{A}_{1} \bigotimes_{F} \widetilde{A}_{2} & \left(\left[\log _{\lambda}\left(1+\frac{\prod_{j=1}^{2}\left(\lambda^{l_{A_{j}}}-1\right)}{\lambda-1}\right),\right.\right. \\
= & \left.\log _{\lambda}\left(1+\frac{\prod_{j=1}^{2}\left(\lambda^{\overline{l_{A_{j}}}}-1\right)}{\lambda-1}\right)\right], \\
& \log _{\lambda}\left(1+\frac{\prod_{j=1}^{2}\left(\lambda^{m_{A_{j}}}-1\right)}{\lambda-1}\right), \\
& {\left[\begin{array}{l}
\log _{\lambda}\left(1+\frac{\prod_{j=1}^{2}\left(\lambda^{r_{A_{j}}}-1\right)}{\lambda-1}\right), \\
\left.\log _{\lambda}\left(1+\frac{\prod_{j=1}^{2}\left(\lambda^{\overline{r_{A_{j}}}}-1\right)}{\lambda-1}\right)\right] .
\end{array}\right.}
\end{aligned}
$$

(3) Multiplication by an ordinary number:

$$
\begin{aligned}
& k_{\cdot}{ }_{F} \widetilde{A}=\left(\left[1-\log _{\lambda}\left(1+\frac{\left(\lambda^{1-\underline{l_{A}}}-1\right)^{k}}{(\lambda-1)^{k-1}}\right),\right.\right. \\
&\left.1-\log _{\lambda}\left(1+\frac{\left(\lambda^{1-\overline{r_{A}}}-1\right)^{k}}{(\lambda-1)^{k-1}}\right)\right], \\
& 1-\log _{\lambda}\left(1+\frac{\left(\lambda^{1-m_{A}}-1\right)^{k}}{(\lambda-1)^{k-1}}\right),
\end{aligned}
$$




$$
\begin{aligned}
& {\left[1-\log _{\lambda}\left(1+\frac{\left(\lambda^{1-\underline{r_{A}}}-1\right)^{k}}{(\lambda-1)^{k-1}}\right),\right.} \\
& \left.\left.1-\log _{\lambda}\left(1+\frac{\left(\lambda^{1-\overline{r_{A}}}-1\right)^{k}}{(\lambda-1)^{k-1}}\right)\right]\right) .
\end{aligned}
$$

(4) Power operation:

$$
\begin{aligned}
& \widetilde{A}^{\wedge_{F} k}=\left(\left[\log _{\lambda}\left(1+\frac{\left(\lambda^{l_{A}}-1\right)^{k}}{(\lambda-1)^{k-1}}\right),\right.\right. \\
& \left.\log _{\lambda}\left(1+\frac{\left(\lambda^{\overline{r_{A}}}-1\right)^{k}}{(\lambda-1)^{k-1}}\right)\right], \\
& \log _{\lambda}\left(1+\frac{\left(\lambda^{m_{A}}-1\right)^{k}}{(\lambda-1)^{k-1}}\right), \\
& {\left[\log _{\lambda}\left(1+\frac{\left(\lambda^{r_{A}}-1\right)^{k}}{(\lambda-1)^{k-1}}\right),\right.} \\
& \left.\left.\log _{\lambda}\left(1+\frac{\left(\lambda^{\overline{r_{A}}}-1\right)^{k}}{(\lambda-1)^{k-1}}\right)\right]\right) .
\end{aligned}
$$

Theorem 5. Let $\widetilde{A}, \widetilde{A}_{1}$, and $\widetilde{A}_{2}$ be three TIT2FNs, and $k, k_{1}$, $k_{2}>0$; then one has

(1) $\widetilde{A}_{1} \bigoplus_{F} \widetilde{A}_{2}=\widetilde{A}_{2} \bigoplus_{F} \widetilde{A_{1}}$;

(2) $k_{F}\left(\widetilde{A_{1}} \bigoplus_{F} \widetilde{A}_{2}\right)=k \cdot{ }_{F} \widetilde{A}_{1} \bigoplus_{F} k \cdot{ }_{F} \widetilde{A}_{2}$;

(3) $k_{1} \cdot{ }_{F} \widetilde{A} \bigoplus_{F} k_{2} \cdot{ }_{F} \widetilde{A}=\left(k_{1}+k_{2}\right) \cdot{ }_{F} \widetilde{A}$;

(4) $\left(k_{1} k_{2}\right) \cdot{ }_{F} \widetilde{A}=k_{1} \cdot{ }_{F}\left(k_{2} \cdot{ }_{F} \widetilde{A}\right)$.

It is easy to prove the formulas in Theorem 5; thus, they are omitted in here.

\section{Frank Aggregation Operators for Triangular Interval Type-2 Fuzzy Numbers}

In this section, we will develop the Frank operator under triangular interval type-2 fuzzy environment and propose the triangular interval type-2 fuzzy Frank weighted averaging (TIT2FFWA) operator and the triangular interval type2 fuzzy Frank weighted geometric (TIT2FFWG) operator based on Frank triangular norms, respectively.

\subsection{Triangular Interval Type-2 Fuzzy Frank Weighted Averaging Operator}

Definition 6. Let $\widetilde{A}_{j}=\left(\left[l_{A_{j}}, \overline{l_{A_{j}}}\right], m_{A_{j}},\left[r_{A_{j}}, \overline{r_{A_{j}}}\right]\right)(j=$ $1,2, \ldots, n)$ be a collection of TIT2FNs, and let TIT2FFWA: $\Omega^{n} \rightarrow \Omega$; if

$$
\begin{aligned}
& \operatorname{TIT} 2 F F W A_{\omega}\left(\widetilde{A}_{1}, \widetilde{A}_{2}, \ldots, \widetilde{A}_{n}\right) \\
& =\omega_{1 \cdot F} \widetilde{A}_{1} \bigoplus_{F} \omega_{2} \cdot{ }_{F} \widetilde{A}_{2} \bigoplus_{F} \cdots \bigoplus_{F} \omega_{j \cdot}{ }_{F} \widetilde{A}_{n},
\end{aligned}
$$

then the function TIT2FFWA is called a triangular interval type-2 fuzzy Frank weighted averaging (TIT2FFWA) operator, where $\omega=\left(\omega_{1}, \omega_{2}, \ldots, \omega_{n}\right)^{T}$ is the weight vector of $\widetilde{A}_{j}(j=1,2, \ldots, n), \omega_{j} \geq 0$, and $\sum_{j=1}^{n} \omega_{j}=1$. In particular, if $\omega=(1 / n, 1 / n, \ldots, 1 / n)^{T}$, then the TIT2FFWA operator is reduced to a triangular interval type-2 fuzzy Frank arithmetic averaging (TIT2FFAA) operator of dimension $n$, which is defined as follows:

$$
\begin{aligned}
& \operatorname{TIT2FFAA}_{\omega}\left(\widetilde{A}_{1}, \widetilde{A}_{2}, \ldots, \widetilde{A}_{n}\right) \\
& =\frac{1}{n} \cdot{ }_{F}\left(\widetilde{A}_{1} \oplus_{F} \widetilde{A}_{2} \oplus_{F} \cdots \oplus_{F} \widetilde{A}_{n}\right) .
\end{aligned}
$$

Based on the Frank operation laws from Theorem 5, we can derive the following Theorem 7 , which shows that the FWA of TIT2 fuzzy sets is also a TIT2 fuzzy set.

Theorem 7. Let $\widetilde{A}_{j}=\left(\left[l_{A_{j}}, \overline{l_{A_{j}}}\right], m_{A_{j}},\left[r_{A_{j}}, \overline{r_{A_{j}}}\right]\right)(j=$ $1,2, \ldots, n)$ be a collection of $\overline{T I T} 2 F N s$; then their aggregated value by TIT2FFWA operator is still a TIT2FN, and

$$
\begin{aligned}
\text { TIT2FFWA }{ }_{\omega}\left(\widetilde{A}_{1}, \widetilde{A}_{2}, \ldots, \widetilde{A}_{n}\right) \\
=\left(\left[1-\log _{\lambda}\left(1+\prod_{j=1}^{n}\left(\lambda^{1-l_{A_{j}}}-1\right)^{\omega_{j}}\right),\right.\right. \\
\left.1-\log _{\lambda}\left(1+\prod_{j=1}^{n}\left(\lambda^{1-\overline{l_{A_{j}}}}-1\right)^{\omega_{j}}\right)\right], \\
1-\log _{\lambda}\left(1+\prod_{j=1}^{n}\left(\lambda^{1-m_{A_{j}}}-1\right)^{\omega_{j}}\right) \\
{\left[\begin{array}{l}
1-\log _{\lambda}\left(1+\prod_{j=1}^{n}\left(\lambda^{1-r_{A_{j}}}-1\right)^{\omega_{j}}\right) \\
\left.1-\log _{\lambda}\left(1+\prod_{j=1}^{n}\left(\lambda^{1-\overline{r_{A_{j}}}}-1\right)^{\omega_{j}}\right)\right],
\end{array}\right.}
\end{aligned}
$$


where $\omega=\left(\omega_{1}, \omega_{2}, \ldots, \omega_{n}\right)^{T}$ is the weight vector of $\widetilde{A}_{j}(j=$ $1,2, \ldots, n), \omega_{j}>0$, and $\sum_{j=1}^{n} \omega_{j}=1$.

Proof. Here, we prove Theorem 7 by using the mathematical induction method as follows.

(1) For $n=2$, based on the Frank operational laws of TIT2FNs, we have

$$
\begin{aligned}
\omega_{1}{ }_{F} \widetilde{A}_{1} & \left(\left[1-\log _{\lambda}\left(1+\frac{\left(\lambda^{1-\underline{l_{A_{1}}}}-1\right)^{\omega_{1}}}{(\lambda-1)^{\omega_{1}-1}}\right),\right.\right. \\
& \left.1-\log _{\lambda}\left(1+\frac{\left(\lambda^{1-\overline{l_{A_{1}}}}-1\right)^{\omega_{1}}}{(\lambda-1)^{\omega_{1}-1}}\right)\right], \\
& 1-\log _{\lambda}\left(1+\frac{\left(\lambda^{\left.1-m_{A_{1}}-1\right)^{\omega_{1}}}\right.}{(\lambda-1)^{\omega_{1}-1}}\right) \\
& {\left[\begin{array}{l}
1-\log _{\lambda}\left(1+\frac{\left(\lambda^{1-r_{A_{1}}}-1\right)^{\omega_{1}}}{(\lambda-1)^{\omega_{1}-1}}\right) \\
1-\log _{\lambda}\left(1+\frac{\left(\lambda^{1-\overline{r_{A_{1}}}}-1\right)^{\omega_{1}}}{(\lambda-1)^{\omega_{1}-1}}\right)
\end{array}\right), }
\end{aligned}
$$

$\omega_{2} \cdot{ }_{F} \widetilde{A}_{2}$

$$
\begin{gathered}
=\left(\left[1-\log _{\lambda}\left(1+\frac{\left(\lambda^{1-l_{A_{2}}}-1\right)^{\omega_{2}}}{(\lambda-1)^{\omega_{2}-1}}\right),\right.\right. \\
\left.1-\log _{\lambda}\left(1+\frac{\left(\lambda^{1-\overline{l_{A_{2}}}}-1\right)^{\omega_{2}}}{(\lambda-1)^{\omega_{2}-1}}\right)\right], \\
1-\log _{\lambda}\left(1+\frac{\left(\lambda^{1-m_{A_{2}}}-1\right)^{\omega_{2}}}{(\lambda-1)^{\omega_{2}-1}}\right), \\
1-\log _{\lambda}\left(1+\frac{\left(\lambda^{1-r_{A_{2}}}-1\right)^{\omega_{2}}}{(\lambda-1)^{\omega_{2}-1}}\right), \\
\left.1-\log _{\lambda}\left(1+\frac{\left(\lambda^{1-\overline{r_{A_{2}}}}-1\right)^{\omega_{2}}}{(\lambda-1)^{\omega_{2}-1}}\right)\right] .
\end{gathered}
$$

$$
\begin{aligned}
& \operatorname{TIT} 2 F F W A_{\omega}\left(\widetilde{A}_{1}, \widetilde{A}_{2}\right) \\
& =\omega_{1} \cdot{ }_{F} \widetilde{A}_{1} \bigoplus_{F} \omega_{2} \cdot{ }_{F} \widetilde{A}_{2} \\
& =\left(\left[1-\log _{\lambda}\left(1+\frac{\prod_{j=1}^{2}\left(\lambda^{1-\left(1-\log _{\lambda}\left(1+\left(\lambda^{1-l_{A_{j}}}-1\right)^{\omega_{j}} /(\lambda-1)^{\omega_{j}-1}\right)\right)}-1\right)}{\lambda-1}\right),\right.\right. \\
& \left.1-\log _{\lambda}\left(1+\frac{\prod_{j=1}^{2}\left(\lambda^{1-\left(1-\log _{\lambda}\left(1+\left(\lambda^{1-\overline{A_{j}}}-1\right)^{\omega_{j}} /(\lambda-1)^{\omega_{j}-1}\right)\right)}-1\right)}{\lambda-1}\right)\right], \\
& 1-\log _{\lambda}\left(1+\frac{\prod_{j=1}^{2}\left(\lambda^{1-\left(1-\log _{\lambda}\left(1+\left(\lambda^{1-m_{A}}-1\right)^{\omega_{j}} /(\lambda-1)^{\omega_{j}-1}\right)\right.}-1\right)}{\lambda-1}\right), \\
& {\left[1-\log _{\lambda}\left(1+\frac{\prod_{j=1}^{2}\left(\lambda^{1-\left(1-\log _{\lambda}\left(1+\left(\lambda^{1-r_{A}}-1\right)^{\omega_{j}} /(\lambda-1)^{\omega_{j}-1}\right)\right)}-1\right)}{\lambda-1}\right),\right.} \\
& \left.\left.1-\log _{\lambda}\left(1+\frac{\prod_{j=1}^{2}\left(\lambda^{1-\left(1-\log _{\lambda}\left(1+\left(\lambda^{1-\bar{r}_{A_{j}}}-1\right)^{\omega_{j}} /(\lambda-1)^{\omega_{j}-1}\right)\right)}-1\right)}{\lambda-1}\right)\right]\right)
\end{aligned}
$$




$$
\begin{aligned}
& =\left(\left[1-\log _{\lambda}\left(1+\frac{\prod_{j=1}^{2}\left(\lambda^{1-l_{A_{j}}}-1\right)^{\omega_{j}}}{(\lambda-1)^{\omega_{1}+\omega_{2}-1}}\right), 1-\log _{\lambda}\left(1+\frac{\prod_{j=1}^{2}\left(\lambda^{1-\overline{l_{A_{j}}}}-1\right)^{\omega_{j}}}{(\lambda-1)^{\omega_{1}+\omega_{2}-1}}\right)\right], 1-\log _{\lambda}\left(1+\frac{\prod_{j=1}^{2}\left(\lambda^{1-m_{A_{j}}}-1\right)^{\omega_{j}}}{(\lambda-1)^{\omega_{1}+\omega_{2}-1}}\right),\right. \\
& =\left(\left[1-\log _{\lambda}\left(1+\frac{\prod_{j=1}^{2}\left(\lambda^{1-r_{A_{j}}}-1\right)^{\omega_{j}}}{(\lambda-1)^{\omega_{1}+\omega_{2}-1}}\right), 1-\log _{\lambda}\left(1+\frac{\prod_{j=1}^{2}\left(\lambda^{1-\overline{r_{A_{j}}}}-1\right)^{\omega_{j}}}{(\lambda-1)^{\omega_{1}+\omega_{2}-1}}\right)\right]\right) \\
& =\left[1-\log _{\lambda}\left(1+\prod_{j=1}^{2}\left(\lambda^{1-l_{A_{j}}}-1\right)^{\omega_{j}}\right), 1-\log _{\lambda}\left(1+\prod_{j=1}^{2}\left(\lambda^{1-\overline{A_{A_{j}}}}-1\right)^{\omega_{j}}\right)\right], 1-\log _{\lambda}\left(1+\prod_{j=1}^{2}\left(\lambda^{1-m_{A_{j}}}-1\right)^{\omega_{j}}\right), \\
& \\
& \left.\left[1-\log _{\lambda}\left(1+\prod_{j=1}^{2}\left(\lambda^{1-r_{A_{j}}}-1\right)^{\omega_{j}}\right), 1-\log _{\lambda}\left(1+\prod_{j=1}^{2}\left(\lambda^{1-\overline{r_{A_{j}}}}-1\right)^{\omega_{j}}\right)\right]\right) .
\end{aligned}
$$

If (15) holds for $n=k$, that is,

$$
\begin{aligned}
& \text { TIT2FFWA }_{\omega}\left(\widetilde{A}_{1}, \widetilde{A}_{2}, \ldots, \widetilde{A}_{k}\right)\left(\left[1-\log _{\lambda}\left(1+\prod_{j=1}^{k}\left(\lambda^{1-l_{A_{j}}}-1\right)^{\omega_{j}}\right),\right.\right. \\
&\left.1-\log _{\lambda}\left(1+\prod_{j=1}^{k}\left(\lambda^{1-\overline{A_{A_{j}}}}-1\right)^{\omega_{j}}\right)\right], \\
& 1-\log _{\lambda}\left(1+\prod_{j=1}^{k}\left(\lambda^{1-m_{A_{j}}}-1\right)^{\omega_{j}}\right), \\
& \quad\left[\begin{array}{ll}
1-\log _{\lambda}\left(1+\prod_{j=1}^{k}\left(\lambda^{1-r_{A_{j}}}-1\right)^{\omega_{j}}\right), \\
\left.\left.1-\log _{\lambda}\left(1+\prod_{j=1}^{k}\left(\lambda^{1-\overline{r_{A_{j}}}}-1\right)^{\omega_{j}}\right)\right]\right),
\end{array}\right.
\end{aligned}
$$

then if $n=k+1$, based on the operational laws of the TIT2FNs, we can deduce that

$$
\begin{aligned}
\text { TIT2FFWA }_{\omega}\left(\widetilde{A}_{1}, \widetilde{A}_{2}, \ldots, \widetilde{A}_{k}, \widetilde{A}_{k+1}\right) \\
=\left(\left[1-\log _{\lambda}\left(1+\prod_{j=1}^{k}\left(\lambda^{1-l_{A_{j}}}-1\right)^{\omega_{j}}\right),\right.\right. \\
\left.1-\log _{\lambda}\left(1+\prod_{j=1}^{k}\left(\lambda^{1-\overline{l_{A_{j}}}}-1\right)^{\omega_{j}}\right)\right], \\
1-\log _{\lambda}\left(1+\prod_{j=1}^{k}\left(\lambda^{1-m_{A_{j}}}-1\right)^{\omega_{j}}\right),
\end{aligned}
$$

$$
\begin{gathered}
{\left[1-\log _{\lambda}\left(1+\prod_{j=1}^{k}\left(\lambda^{1-r_{A_{j}}}-1\right)^{\omega_{j}}\right),\right.} \\
\left.1-\log _{\lambda}\left(1+\prod_{j=1}^{k}\left(\lambda^{1-\overline{r_{A_{j}}}}-1\right)^{\omega_{j}}\right)\right] \\
\bigoplus_{F}\left(\left[1-\log _{\lambda}\left(1+\frac{\left(\lambda^{1-l_{A_{k+1}}}-1\right)^{\omega_{k+1}}}{(\lambda-1)^{\omega_{k+1}-1}}\right),\right.\right. \\
\left.1-\log _{\lambda}\left(1+\frac{\left(\lambda^{1-\overline{l_{A_{k+1}}}}-1\right)^{\omega_{k+1}}}{(\lambda-1)^{\omega_{k+1}-1}}\right)\right], \\
1-\log _{\lambda}\left(1+\frac{\left(\lambda^{1-m_{A_{k+1}}}-1\right)^{\omega_{k+1}}}{(\lambda-1)^{\omega_{k+1}-1}}\right), \\
{\left[1-\log _{\lambda}\left(1+\frac{\left(\lambda^{1-r_{A_{k+1}}}-1\right)^{\omega_{k+1}}}{(\lambda-1)^{\omega_{k+1}-1}}\right),\right.}
\end{gathered}
$$$$
\left.\left.1-\log _{\lambda}\left(1+\frac{\left(\lambda^{1-\overline{r_{A_{k+1}}}}-1\right)^{\omega_{k+1}}}{(\lambda-1)^{\omega_{k+1}-1}}\right)\right]\right)
$$$$
=\left(\left[1-\log _{\lambda}\left(1+\frac{\prod_{j=1}^{k+1}\left(\lambda^{1-l_{A_{j}}}-1\right)^{\omega_{j}}}{(\lambda-1)^{\sum_{j=1}^{k+1} \omega_{j}-1}}\right),\right.\right.
$$$$
\left.1-\log _{\lambda}\left(1+\frac{\prod_{j=1}^{k+1}\left(\lambda^{1-\overline{l_{A_{j}}}}-1\right)^{\omega_{j}}}{(\lambda-1)^{\sum_{j=1}^{k+1} \omega_{j}-1}}\right)\right],
$$$$
1-\log _{\lambda}\left(1+\frac{\prod_{j=1}^{k+1}\left(\lambda^{1-m_{A_{j}}}-1\right)^{\omega_{j}}}{(\lambda-1)^{\sum_{j=1}^{k+1} \omega_{j}-1}}\right),
$$ 


$$
\begin{aligned}
& {\left[1-\log _{\lambda}\left(1+\frac{\prod_{j=1}^{k+1}\left(\lambda^{1-r_{A_{j}}}-1\right)^{\omega_{j}}}{(\lambda-1)^{\sum_{j=1}^{k+1} \omega_{j}-1}}\right),\right.} \\
& \left.\left.1-\log _{\lambda}\left(1+\frac{\prod_{j=1}^{k+1}\left(\lambda^{1-\overline{A_{A_{j}}}}-1\right)^{\omega_{j}}}{(\lambda-1)^{\sum_{j=1}^{k+1} \omega_{j}-1}}\right)\right]\right) \\
=( & 11-\log _{\lambda}\left(1+\prod_{j=1}^{k+1}\left(\lambda^{1-l_{A_{j}}}-1\right)^{\omega_{j}}\right), \\
& \left.1-\log _{\lambda}\left(1+\prod_{j=1}^{k+1}\left(\lambda^{1-\overline{l_{A_{j}}}}-1\right)^{\omega_{j}}\right)\right], \\
& 1-\log _{\lambda}\left(1+\prod_{j=1}^{k+1}\left(\lambda^{1-m_{A_{j}}}-1\right)^{\omega_{j}}\right), \\
& {\left[1-\log _{\lambda}\left(1+\prod_{j=1}^{k+1}\left(\lambda^{1-r_{A_{j}}}-1\right)^{\omega_{j}}\right),\right.} \\
& \left.1-\log _{\lambda}\left(1+\prod_{j=1}^{k+1}\left(\lambda^{1-\overline{r_{A_{j}}}}-1\right)^{\omega_{j}}\right)\right] .
\end{aligned}
$$

That is, (15) holds for $n=k+1$. Therefore, (15) holds for all $n$, which completes the proof of Theorem 7 .

In what follows, we can easily prove that the TIT2FFWA operator has the idempotency, boundary, and other properties that ordinary aggregation operators usually have.

Theorem 8 (idempotency). Let $\widetilde{A}_{j}=\left(\left[l_{A_{j}}, \overline{l_{A_{j}}}\right], m_{A_{j}}\right.$, $\left.\left[r_{A_{j}}, \overline{r_{A_{j}}}\right]\right)(j=1,2, \ldots, n)$ be a collection of TIT2FNs, where $\overline{0} \leq l_{A_{j}} \leq \overline{l_{A_{j}}} \leq m_{A_{j}} \leq r_{A_{j}} \leq \overline{r_{A_{j}}} \leq 1$. If all $\widetilde{A}_{j} \quad(j=$ $1,2, \ldots, n)$ are equal, that is, $\overline{\widetilde{A}_{j}}=\widetilde{A}$, for all $j$, then

$$
\operatorname{TIT} 2 F F W A_{\omega}\left(\widetilde{A}_{1}, \widetilde{A}_{2}, \ldots, \widetilde{A}_{n}\right)=\widetilde{A} .
$$

Proof. By using Theorem 7, we have

$$
\begin{aligned}
\text { TIT2FFWA }_{\omega}\left(\widetilde{A}_{1}, \widetilde{A}_{2}, \ldots, \widetilde{A}_{n}\right) & \left(\left[1-\log _{\lambda}\left(1+\left(\lambda^{1-l_{A}}-1\right)^{\sum_{j=1}^{n} \omega_{j}}\right),\right.\right. \\
& \left.1-\log _{\lambda}\left(1+\left(\lambda^{1-\overline{l_{A}}}-1\right)^{\sum_{j=1}^{n} \omega_{j}}\right)\right], \\
& 1-\log _{\lambda}\left(1+\left(\lambda^{1-m_{A}}-1\right)^{\sum_{j=1}^{n} \omega_{j}}\right), \\
& {\left[1-\log _{\lambda}\left(1+\left(\lambda^{1-r_{A_{j}}}-1\right)^{\sum_{j=1}^{n} \omega_{j}}\right),\right.} \\
& \left.\left.1-\log _{\lambda}\left(1+\left(\lambda^{1-\overline{r_{A_{j}}}}-1\right)^{\sum_{j=1}^{n} \omega_{j}}\right)\right]\right)
\end{aligned}
$$

$$
\begin{aligned}
= & \left(\left[1-\log _{\lambda} \lambda^{1-l_{\underline{A}}}, 1-\log _{\lambda} \lambda^{1-\overline{l_{A}}}\right], 1-\log _{\lambda} \lambda^{1-m_{A}},\right. \\
& {\left.\left[1-\log _{\lambda} \lambda^{1-\underline{r_{A_{j}}}}, 1-\log _{\lambda} \lambda^{1-\overline{r_{A_{j}}}}\right]\right) } \\
= & \left(\left[1-\left(1-\underline{l_{A}}\right), 1-\left(1-\overline{l_{A}}\right)\right], 1-\left(1-m_{A}\right),\right. \\
& \left.\quad\left[1-\left(1-\underline{r_{A_{j}}}\right), 1-\left(1-\overline{r_{A_{j}}}\right)\right]\right) \\
= & \left.\left(\left[\underline{l_{A}}, \overline{l_{A}}\right], m_{A}, \underline{r_{A}}, \overline{r_{A}}\right]\right)=\widetilde{A} .
\end{aligned}
$$

Thus, the proof is completed.

Theorem 9 (boundary). Let $\widetilde{A}_{j}=\left(\left[l_{A_{j}}, \overline{l_{A_{j}}}\right]\right.$, $\left.m_{A_{j}},\left[r_{A_{j}}, \overline{r_{A_{j}}}\right]\right)(j=1,2, \ldots, n)$ be a collection of TIT2FNs, where $\overline{0} \leq l_{A_{j}} \leq \overline{l_{A_{j}}} \leq m_{A_{j}} \leq r_{A_{j}} \leq \overline{r_{A_{j}}} \leq 1$, and let

$$
\begin{aligned}
& \widetilde{A}^{+}=\{ {\left[\max _{j=1}^{n}\left\{l_{A_{j}}\right\}, \max _{j=1}^{n}\left\{\overline{l_{A_{j}}}\right\}\right], \max _{j=1}^{n}\left\{m_{A_{j}}\right\}, } \\
& {\left.\left[\max _{j=1}^{n}\left\{\underline{r_{A_{j}}}\right\}, \max _{j=1}^{n}\left\{\overline{r_{A_{j}}}\right\}\right]\right\}, } \\
& \widetilde{A}^{-}=\left\{\left[\min _{j=1}^{n}\left\{\underline{l_{A_{j}}}\right\}, \min _{j=1}^{n}\left\{\overline{l_{A_{j}}}\right\}\right], \min _{j=1}^{n}\left\{m_{A_{j}}\right\},\right. \\
& {\left.\left[\min _{j=1}^{n}\left\{\underline{r_{A_{j}}}\right\}, \min _{j=1}^{n}\left\{\overline{r_{A_{j}}}\right\}\right]\right\} . }
\end{aligned}
$$

Then

$$
\widetilde{A}^{-} \leq \operatorname{TIT2FFWA}\left(\widetilde{A}_{1}, \widetilde{A}_{2}, \ldots, \widetilde{A}_{n}\right) \leq \widetilde{A}^{+} .
$$

Proof. Since

$$
\begin{gathered}
\min \left\{\underline{\left.l_{A_{j}}\right\}} \leq \underline{\underline{A_{j}}} \leq \max \left\{\underline{l_{A_{j}}}\right\},\right. \\
\min _{j}\left\{\overline{\overline{l_{A_{j}}}}\right\} \leq \overline{\overline{l_{A_{j}}}} \leq \max _{j}\left\{\overline{\overline{l_{A_{j}}}}\right\}, \\
\min _{j}\left\{m_{A_{j}}\right\} \leq m_{A_{j}} \leq \max _{j}\left\{m_{A_{j}}\right\}, \\
\min \left\{\underline{\left.r_{A_{j}}\right\}} \leq \overline{r_{A_{j}}} \leq \max \left\{\underline{r_{A_{j}}}\right\},\right. \\
\min _{j}\left\{\overline{r_{A_{j}}}\right\} \leq \overline{r_{A_{j}}} \leq \max _{j}\left\{\overline{r_{A_{j}}}\right\}
\end{gathered}
$$

for all $j$, then it follows that

$$
1-\max _{j}\left\{\underline{l_{A_{j}}}\right\} \leq 1-\underline{l_{A_{j}}} \leq 1-\min _{j}\left\{\underline{l_{A_{j}}}\right\}
$$

and then

$$
\begin{aligned}
\log _{\lambda}\left(1+\prod_{j=1}^{n}\left(\lambda^{1-\max _{j}\left\{l_{A_{j}}\right\}}-1\right)^{\omega_{j}}\right) \\
\leq \log _{\lambda}\left(1+\prod_{j=1}^{n}\left(\lambda^{1-l_{A_{j}}}-1\right)^{\omega_{j}}\right) \\
\leq \log _{\lambda}\left(1+\prod_{j=1}^{n}\left(\lambda^{1-\min _{j}\left\{l_{A_{j}}\right\}}-1\right)^{\omega_{j}}\right) .
\end{aligned}
$$


Then, it follows that

$$
\begin{aligned}
& \log _{\lambda}\left(1+\left(\lambda^{1-\max _{j}\left\{l_{A_{j}}\right\}}-1\right)^{\sum_{j=1}^{n} \omega_{j}}\right) \\
& \leq \log _{\lambda}\left(1+\prod_{j=1}^{n}\left(\lambda^{1-l_{A_{j}}}-1\right)^{\omega_{j}}\right) \\
& \leq \log _{\lambda}\left(1+\left(\lambda \underline{1-\min _{j}\left\{l_{A_{j}}\right\}}-1\right)^{\sum_{j=1}^{n} \omega_{j}}\right) \\
& \Longrightarrow \log _{\lambda} \lambda^{1-\max _{j}\left\{l_{A_{j}}\right\}} \\
& \leq \log _{\lambda}\left(1+\prod_{j=1}^{n}\left(\lambda^{1-l_{A_{j}}}-1\right)^{\omega_{j}}\right) \leq \log _{\lambda} \lambda \stackrel{1-\min _{j}\left\{l_{A_{j}}\right\}}{ } \\
& \Longrightarrow 1-\max _{j}\left\{\underline{l_{A_{j}}}\right\} \leq \log _{\lambda}\left(1+\prod_{j=1}^{n}\left(\lambda^{1-l_{A_{j}}}-1\right)^{\omega_{j}}\right) \\
& \leq 1-\min _{j}\left\{\underline{l_{A_{j}}}\right\} \\
& \Longrightarrow \min _{j}\left\{\underline{l_{A_{j}}}\right\} \leq 1-\log _{\lambda}\left(1+\prod_{j=1}^{n}\left(\lambda^{1-l_{A_{j}}}-1\right)^{\omega_{j}}\right) \\
& \leq \max _{j}\left\{\underline{l_{A_{j}}}\right\} .
\end{aligned}
$$

Similarly, we have

$$
\begin{aligned}
\min _{j}\left\{\overline{l_{A_{j}}}\right\} & \leq 1-\log _{\lambda}\left(1+\prod_{j=1}^{n}\left(\lambda^{1-\overline{l_{A_{j}}}}-1\right)^{\omega_{j}}\right) \\
& \leq \max _{j}\left\{\overline{l_{A_{j}}}\right\}, \\
\min _{j}\left\{m_{A_{j}}\right\} & \leq 1-\log _{\lambda}\left(1+\prod_{j=1}^{n}\left(\lambda^{1-m_{A_{j}}}-1\right)^{\omega_{j}}\right) \\
& \leq \max _{j}\left\{m_{A_{j}}\right\}, \\
\min _{j}\left\{\underline{r_{A_{j}}}\right\} & \leq 1-\log _{\lambda}\left(1+\prod_{j=1}^{n}\left(\lambda^{1-r_{A_{j}}}-1\right)^{\omega_{j}}\right) \\
& \leq \max _{j}\left\{\underline{r_{A_{j}}}\right\}, \\
\min _{j}\left\{\overline{r_{A_{j}}}\right\} & \leq 1-\log _{\lambda}\left(1+\prod_{j=1}^{n}\left(\lambda^{1-\overline{r_{A_{j}}}}-1\right)^{\omega_{j}}\right) \\
& \leq \max _{j}\left\{\overline{r_{A_{j}}}\right\} .
\end{aligned}
$$

Let TIT2FFWA ${ }_{\omega}\left(\widetilde{A}_{1}, \widetilde{A}_{2}, \ldots, \widetilde{A}_{n}\right)=\widetilde{A}=\left(\left[\underline{l_{A}}, \overline{l_{A}}\right]\right.$, $\left.m_{A},\left[r_{A}, \overline{r_{A}}\right]\right)$; then by using the ranking value formula of TIT2 $2 \overline{\mathrm{FN}}$, we have

$$
\begin{aligned}
& \operatorname{Rank}(\widetilde{A})=\left(\frac{\underline{l_{A}}+\overline{r_{A}}}{2}+1\right) \times \frac{\underline{\underline{l_{A}}+\overline{l_{A}}+\underline{r_{A}}+\overline{r_{A}}+4 m_{A}}}{8} \\
& \leq\left(\frac{\max _{j}\left(\underline{l_{A_{j}}}\right)+\max _{j}\left(\overline{r_{A_{j}}}\right)}{2}+1\right) \\
& \times\left(\max _{j}\left(\underline{l_{A_{j}}}\right)+\max _{j}\left(\overline{l_{A_{j}}}\right)+\max _{j}\left(\underline{r_{A_{j}}}\right)\right. \\
& \left.+\max _{j}\left(\overline{r_{A_{j}}}\right)+4 \max _{j}\left(m_{A_{j}}\right)\right) \times(8)^{-1} \\
& =\operatorname{Rank}\left(\widetilde{A}^{+}\right) \text {, } \\
& \operatorname{Rank}(\widetilde{A})=\left(\frac{l_{A}+\overline{r_{A}}}{2}+1\right) \times \frac{\underline{l_{A}+\overline{l_{A}}+\underline{r_{A}}+\overline{r_{A}}+4 m_{A}}}{8} \\
& \geq\left(\frac{\min _{j}\left(\frac{l_{A_{j}}}{}\right)+\min _{j}\left(\overline{r_{A_{j}}}\right)}{2}+1\right) \\
& \times\left(\min _{j}\left(\underline{l_{A_{j}}}\right)+\min _{j}\left(\overline{l_{A_{j}}}\right)+\min \left(\underline{r_{A_{j}}}\right)\right. \\
& \left.+\min _{j}\left(\overline{r_{A_{j}}}\right)+4 \min _{j}\left(m_{A_{j}}\right)\right) \times(8)^{-1} \\
& =\operatorname{Rank}\left(\widetilde{A}^{-}\right) \text {. }
\end{aligned}
$$

Therefore,

$$
\widetilde{A}^{-} \leq \operatorname{TIT}_{2} \mathrm{FFWA}_{\omega}\left(\widetilde{A}_{1}, \widetilde{A}_{2}, \ldots, \widetilde{A}_{n}\right) \leq \widetilde{A}^{+}
$$

which completes the proof of Theorem 9.

Theorem 10. Let $\widetilde{A}_{j}=\left(\left[l_{A_{j}}, \overline{l_{A_{j}}}\right], m_{A_{j}},\left[r_{A_{j}}, \overline{r_{A_{j}}}\right]\right)(j=$ $1,2, \ldots, n)$ be a collection of TIT2FNs, where $0 \leq l_{A_{j}} \leq$ $\overline{l_{A_{j}}} \leq m_{A_{j}} \leq r_{A_{j}} \leq \overline{r_{A_{j}}} \leq$ 1. If $\widetilde{A_{n+1}}=$ $\left(\left[\underline{l_{A_{n+1}}}, \overline{l_{A_{n+1}}}\right], m_{A_{n+1}},\left[\underline{r_{A_{n+1}}}, \overline{r_{A_{n+1}}}\right]\right)$ is also a TIT2FN on $X$, then

$$
\begin{gathered}
\operatorname{TIT2FFWA}_{\omega}\left(\widetilde{A}_{1} \bigoplus_{F} \widetilde{A}_{n+1}, \widetilde{A}_{2} \bigoplus_{F} \widetilde{A}_{n+1}, \ldots, \widetilde{A}_{n} \bigoplus_{F} \widetilde{A}_{n+1}\right) \\
=\operatorname{TIT} 2 F F W A_{\omega}\left(\widetilde{A}_{1}, \widetilde{A}_{2}, \ldots, \widetilde{A}_{n}\right) \bigoplus_{F} \widetilde{A}_{n+1} .
\end{gathered}
$$


Proof. Since

$$
\begin{aligned}
\widetilde{A}_{j} \bigoplus_{F} \widetilde{A}_{n+1} & \left(\left[1-\log _{\lambda}\left(1+\frac{\prod_{i=\{j, n+1\}}\left(\lambda^{1-l_{A_{i}}}-1\right)}{\lambda-1}\right),\right.\right. \\
= & \left.1-\log _{\lambda}\left(1+\frac{\prod_{i=\{j, n+1\}}\left(\lambda^{1-\overline{l_{A_{i}}}}-1\right)}{\lambda-1}\right)\right], \\
& 1-\log _{\lambda}\left(1+\frac{\prod_{i=\{j, n+1\}}\left(\lambda^{1-m_{A_{i}}}-1\right)}{\lambda-1}\right),
\end{aligned}
$$

$$
\begin{aligned}
& {\left[1-\log _{\lambda}\left(1+\frac{\prod_{i=\{j, n+1\}}\left(\lambda^{1-r_{A_{j}}}-1\right)}{\lambda-1}\right),\right.} \\
& \left.1-\log _{\lambda}\left(1+\frac{\prod_{i=\{j, n+1\}}\left(\lambda^{1-\overline{r_{j}}}-1\right)}{\lambda-1}\right)\right],
\end{aligned}
$$

according to Theorem 7, we have

$$
\begin{aligned}
& \operatorname{TIT2FFAA}_{\omega}\left(\widetilde{A}_{1} \bigoplus_{F} \widetilde{A}_{n+1}, \widetilde{A}_{2} \bigoplus_{F} \widetilde{A}_{n+1}, \ldots, \widetilde{A}_{n} \bigoplus_{F} \widetilde{A}_{n+1}\right) \\
& =\left(\left[1-\log _{\lambda}\left(1+\prod_{j=1}^{n}\left(\lambda^{1-\left(1-\log _{\lambda}\left(1+\prod_{i=\{j, j+1\}}\left(\lambda^{1-l_{A_{i}}}-1\right) /(\lambda-1)\right)\right)}-1\right)^{\omega_{j}}\right)\right.\right. \text {, } \\
& \left.1-\log _{\lambda}\left(1+\prod_{j=1}^{n}\left(\lambda^{1-\left(1-\log _{\lambda}\left(1+\prod_{i=\{j, j+1\}}\left(\lambda^{1-\overline{I_{A_{i}}}}-1\right) /(\lambda-1)\right)\right)}-1\right)^{\omega_{j}}\right)\right],
\end{aligned}
$$

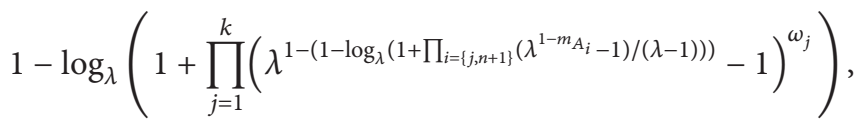

$$
\begin{aligned}
& {\left[1-\log _{\lambda}\left(1+\prod_{j=1}^{k}\left(\lambda^{1-\left(1-\log _{\lambda}\left(1+\prod_{i=\{j, n+1\}}\left(\lambda^{1-r_{A_{i}}}-1\right) /(\lambda-1)\right)\right)}-1\right)^{\omega_{j}}\right),\right.} \\
& \left.\left.1-\log _{\lambda}\left(1+\prod_{j=1}^{k}\left(\lambda^{1-\left(1-\log _{\lambda}\left(1+\prod_{i=\{j, n+1\}}\left(\lambda^{1-\overline{\digamma_{A}}}-1\right) /(\lambda-1)\right)\right)}-1\right)^{\omega_{j}}\right)\right]\right) \\
& =\left(\left[1-\log _{\lambda}\left(1+\frac{\prod_{j=1}^{n}\left(\lambda^{1-l_{A_{j}}}-1\right)^{\omega_{j}}\left(\lambda^{1-l_{A_{n+1}}}-1\right)^{\sum_{j=1}^{n} \omega_{j}}}{\lambda-1}\right), 1-\log _{\lambda}\left(1+\frac{\prod_{j=1}^{n}\left(\lambda^{1-\overline{l_{A_{j}}}}-1\right)^{\omega_{j}}\left(\lambda^{1-\overline{l_{A_{n+1}}}}-1\right)^{\sum_{j=1}^{n} \omega_{j}}}{\lambda-1}\right)\right]\right. \text {, } \\
& 1-\log _{\lambda}\left(1+\frac{\prod_{j=1}^{n}\left(\lambda^{1-m_{A_{j}}}-1\right)^{\omega_{j}}\left(\lambda^{1-m_{A_{n+1}}}-1\right)^{\sum_{j=1}^{n} \omega_{j}}}{\lambda-1}\right), \\
& \left.\left[1-\log _{\lambda}\left(1+\frac{\prod_{j=1}^{n}\left(\lambda^{1-r_{A_{j}}}-1\right)^{\omega_{j}}\left(\lambda^{1-r_{A_{n+1}}}-1\right)^{\sum_{j=1}^{n} \omega_{j}}}{\lambda-1}\right), 1-\log _{\lambda}\left(1+\frac{\prod_{j=1}^{n}\left(\lambda^{1-\overline{r_{A_{j}}}}-1\right)^{\omega_{j}}\left(\lambda^{1-\overline{r_{A_{n+1}}}}-1\right)^{\sum_{j=1}^{n} \omega_{j}}}{\lambda-1}\right)\right]\right) \\
& =\left(\left[1-\log _{\lambda}\left(1+\frac{\prod_{j=1}^{n}\left(\lambda^{1-l_{A_{j}}}-1\right)^{\omega_{j}}\left(\lambda^{1-l_{A_{n+1}}}-1\right)}{\lambda-1}\right), 1-\log _{\lambda}\left(1+\frac{\prod_{j=1}^{n}\left(\lambda^{1-\overline{l_{A_{j}}}}-1\right)^{\omega_{j}}\left(\lambda^{1-\overline{l_{A_{n+1}}}}-1\right)}{\lambda-1}\right)\right],\right.
\end{aligned}
$$




$$
\begin{aligned}
& 1-\log _{\lambda}\left(1+\frac{\prod_{j=1}^{n}\left(\lambda^{1-m_{A_{j}}}-1\right)^{\omega_{j}}\left(\lambda^{1-m_{A_{n+1}}}-1\right)}{\lambda-1}\right), \\
& {\left[1-\log _{\lambda}\left(1+\frac{\prod_{j=1}^{n}\left(\lambda^{1-r_{A_{j}}}-1\right)^{\omega_{j}}\left(\lambda^{1-r_{A_{n+1}}}-1\right)}{\lambda-1}\right), 1-\log _{\lambda}\left(1+\frac{\prod_{j=1}^{n}\left(\lambda^{1-\overline{A_{A_{j}}}}-1\right)^{\omega_{j}}\left(\lambda^{1-\overline{r_{A_{n+1}}}}-1\right)}{\lambda-1}\right)\right] .}
\end{aligned}
$$

On the other hand, based on Theorem 7 and the operational laws of TIT2FNs, we have

$$
\begin{aligned}
& \operatorname{TIT2FFAA}_{\omega}\left(\widetilde{A}_{1}, \widetilde{A}_{2}, \ldots, \widetilde{A}_{n}\right) \bigoplus_{F} \widetilde{A}_{n+1} \\
& =\left(\left[1-\log _{\lambda}\left(1+\prod_{j=1}^{n}\left(\lambda^{1-l_{A_{j}}}-1\right)^{\omega_{j}}\right), 1-\log _{\lambda}\left(1+\prod_{j=1}^{n}\left(\lambda^{1-\overline{l_{A_{j}}}}-1\right)^{\omega_{j}}\right)\right]\right. \\
& 1-\log _{\lambda}\left(1+\prod_{j=1}^{n}\left(\lambda^{1-m_{A_{j}}}-1\right)^{\omega_{j}}\right) \\
& \left.\left[1-\log _{\lambda}\left(1+\prod_{j=1}^{n}\left(\lambda^{1-r_{A_{j}}}-1\right)^{\omega_{j}}\right), 1-\log _{\lambda}\left(1+\prod_{j=1}^{n}\left(\lambda^{1-\overline{r_{A_{j}}}}-1\right)^{\omega_{j}}\right)\right]\right) \\
& \bigoplus_{F}\left(\left[\underline{l_{A_{n+1}}}, \overline{l_{A_{n+1}}}\right], m_{A_{n+1}},\left[\underline{r_{A_{n+1}}}, \overline{r_{A_{n+1}}}\right]\right) \\
& =\left(\left[1-\log _{\lambda}\left(1+\frac{\prod_{j=1}^{n}\left(\lambda^{1-l_{A_{j}}}-1\right)^{\omega_{j}}\left(\lambda^{1-l_{A_{n+1}}}-1\right)}{\lambda-1}\right), 1-\log _{\lambda}\left(1+\frac{\prod_{j=1}^{n}\left(\lambda^{1-\overline{l_{A_{j}}}}-1\right)^{\omega_{j}}\left(\lambda^{1-\overline{l_{A_{n+1}}}}-1\right)}{\lambda-1}\right)\right],\right. \\
& 1-\log _{\lambda}\left(1+\frac{\prod_{j=1}^{n}\left(\lambda^{1-m_{A_{j}}}-1\right)^{\omega_{j}}\left(\lambda^{1-m_{A_{n+1}}}-1\right)}{\lambda-1}\right), \\
& \left.\left[1-\log _{\lambda}\left(1+\frac{\prod_{j=1}^{n}\left(\lambda^{1-r_{A_{j}}}-1\right)^{\omega_{j}}\left(\lambda^{1-r_{A_{n+1}}}-1\right)}{\lambda-1}\right), 1-\log _{\lambda}\left(1+\frac{\prod_{j=1}^{n}\left(\lambda^{1-\overline{r_{A_{j}}}}-1\right)^{\omega_{j}}\left(\lambda^{1-\overline{r_{A_{n+1}}}}-1\right)}{\lambda-1}\right)\right]\right) .
\end{aligned}
$$

Therefore, we have

$$
\begin{gathered}
{\operatorname{TIT} 2 F^{\prime}}_{\omega}\left(\widetilde{A}_{1} \bigoplus_{F} \widetilde{A}_{n+1}, \widetilde{A}_{2} \bigoplus_{F} \widetilde{A}_{n+1}, \ldots, \widetilde{A}_{n} \bigoplus_{F} \widetilde{A}_{n+1}\right) \\
=\operatorname{TIT2FFWA}_{\omega}\left(\widetilde{A}_{1}, \widetilde{A}_{2}, \ldots, \widetilde{A}_{n}\right) \bigoplus_{F} \widetilde{A}_{n+1}
\end{gathered}
$$

which completes the proof of Theorem 10.
Theorem 11. Let $\widetilde{A}_{j}=\left(\left[l_{A_{j}}, \overline{l_{A_{j}}}\right], m_{A_{j}},\left[r_{A_{j}}, \overline{r_{A_{j}}}\right]\right)(j=$ $1,2, \ldots, n)$ be a collection of TIT2FNs, where $\overline{0} \leq l_{A_{j}} \leq \overline{l_{A_{j}}} \leq$ $m_{A_{j}} \leq \underline{r_{A_{j}}} \leq \overline{r_{A_{j}}} \leq 1$. If $r>0$, then

$$
\begin{aligned}
& \operatorname{TIT2FFWA}_{\omega}\left(r_{{ }_{F}} \widetilde{A}_{1}, r_{F} \widetilde{A}_{2}, \ldots, r_{F} \widetilde{A}_{n}\right) \\
& =r \cdot{ }_{F} T I T 2 F F W A_{\omega}\left(\widetilde{A}_{1}, \widetilde{A}_{2}, \ldots, \widetilde{A}_{n}\right) .
\end{aligned}
$$


Proof. Based on the Frank operation laws of TIT2FNs, we have

$r \cdot{ }_{F} \widetilde{A}_{j}$

$$
\begin{gathered}
=\left(\left[1-\log _{\lambda}\left(1+\frac{\left(\lambda^{1-l_{A_{j}}}-1\right)^{r}}{(\lambda-1)^{r-1}}\right), 1-\log _{\lambda}\left(1+\frac{\left(\lambda^{1-\overline{A_{A_{j}}}}-1\right)^{r}}{(\lambda-1)^{r-1}}\right)\right], 1-\log _{\lambda}\left(1+\frac{\left(\lambda^{1-m_{A_{j}}}-1\right)^{r}}{(\lambda-1)^{r-1}}\right),\right. \\
{\left[1-\log _{\lambda}\left(1+\frac{\left(\lambda^{1-r_{A_{j}}}-1\right)^{r}}{(\lambda-1)^{r-1}}\right), 1-\log _{\lambda}\left(1+\frac{\left(\lambda^{1-\overline{r_{A_{j}}}}-1\right)^{r}}{(\lambda-1)^{r-1}}\right)\right],}
\end{gathered}
$$

$\operatorname{TIT} 2 F F A A_{\omega}\left(r_{{ }_{F}} \widetilde{A}_{1}, r_{F} \widetilde{A}_{2}, \ldots, r r_{F} \widetilde{A}_{n}\right)$

$$
\begin{aligned}
& =\left(\left[1-\log _{\lambda}\left(1+\prod_{j=1}^{k}\left(\lambda^{1-\left(1-\log _{\lambda}\left(1+\left(\lambda^{1-l_{A_{j}}}-1\right)^{r} /(\lambda-1)^{r-1}\right)\right)}-1\right)^{\omega_{j}}\right), 1-\log _{\lambda}\left(1+\prod_{j=1}^{k}\left(\lambda^{1-\left(1-\log _{\lambda}\left(1+\left(\lambda^{1-\overline{A_{A_{j}}}}-1\right)^{r} /(\lambda-1)^{r-1}\right)\right)}-1\right)^{\omega_{j}}\right)\right],\right. \\
& 1-\log _{\lambda}\left(1+\prod_{j=1}^{k}\left(\lambda^{1-\left(1-\log _{\lambda}\left(1+\left(\lambda^{1-m_{A_{j}}}-1\right)^{r} /(\lambda-1)^{r-1}\right)\right)}-1\right)^{\omega_{j}}\right), \\
& \left.\left[1-\log _{\lambda}\left(1+\prod_{j=1}^{k}\left(\lambda^{1-\left(1-\log _{\lambda}\left(1+\left(\lambda^{1-r_{A_{j}}}-1\right)^{r} /(\lambda-1)^{r-1}\right)\right)}-1\right)^{\omega_{j}}\right), 1-\log _{\lambda}\left(1+\prod_{j=1}^{k}\left(\lambda^{1-\left(1-\log _{\lambda}\left(1+\left(\lambda^{1-\overline{r_{A_{j}}}}-1\right)^{r} /(\lambda-1)^{r-1}\right)\right)}-1\right)^{\omega_{j}}\right)\right]\right) \\
& =\left(\left[1-\log _{\lambda}\left(1+\frac{\prod_{j=1}^{k}\left(\lambda^{1-l_{A_{j}}}-1\right)^{r \omega_{j}}}{(\lambda-1)^{r-1}}\right), 1-\log _{\lambda}\left(1+\frac{\prod_{j=1}^{k}\left(\lambda^{1-\overline{l_{A_{j}}}}-1\right)^{r \omega_{j}}}{(\lambda-1)^{r-1}}\right)\right], 1-\log _{\lambda}\left(1+\frac{\prod_{j=1}^{k}\left(\lambda^{1-m_{A_{j}}}-1\right)^{r \omega_{j}}}{(\lambda-1)^{r-1}}\right)\right. \text {, } \\
& \left.\left[1-\log _{\lambda}\left(1+\frac{\prod_{j=1}^{k}\left(\lambda^{1-r_{A_{j}}}-1\right)^{r \omega_{j}}}{(\lambda-1)^{r-1}}\right), 1-\log _{\lambda}\left(1+\frac{\prod_{j=1}^{k}\left(\lambda^{1-\overline{r_{A_{j}}}}-1\right)^{r \omega_{j}}}{(\lambda-1)^{r-1}}\right)\right]\right) .
\end{aligned}
$$

Also since

$$
\begin{aligned}
r_{F}{ }_{F} \text { TIT2 } & \text {FFWA }_{\omega}\left(\widetilde{A}_{1}, \widetilde{A}_{2}, \ldots, \widetilde{A}_{n}\right) \\
=r_{F}( & {\left[1-\log _{\lambda}\left(1+\prod_{j=1}^{n}\left(\lambda^{1-l_{A_{j}}}-1\right)^{\omega_{j}}\right), 1-\log _{\lambda}\left(1+\prod_{j=1}^{n}\left(\lambda^{1-\overline{l_{A_{j}}}}-1\right)^{\omega_{j}}\right)\right], } \\
& 1-\log _{\lambda}\left(1+\prod_{j=1}^{n}\left(\lambda^{1-m_{A_{j}}}-1\right)^{\omega_{j}}\right), \\
& {\left.\left[1-\log _{\lambda}\left(1+\prod_{j=1}^{n}\left(\lambda^{1-r_{A_{j}}}-1\right)^{\omega_{j}}\right), 1-\log _{\lambda}\left(1+\prod_{j=1}^{n}\left(\lambda^{1-\overline{r_{A_{j}}}}-1\right)^{\omega_{j}}\right)\right]\right) }
\end{aligned}
$$




$$
\begin{aligned}
& =\left(\left[1-\log _{\lambda}\left(1+\frac{\left(\lambda^{1-\left(1-\log _{\lambda}\left(1+\prod_{j=1}^{n}\left(\lambda^{1-l_{A_{j}}}-1\right)^{\omega_{j}}\right)\right)}-1\right)^{r}}{(\lambda-1)^{r-1}}\right), 1-\log _{\lambda}\left(1+\frac{\left(\lambda^{1-\left(1-\log _{\lambda}\left(1+\prod_{j=1}^{n}\left(\lambda^{1-\overline{l_{A_{j}}}}-1\right)^{\omega_{j}}\right)\right)}-1\right)^{r}}{(\lambda-1)^{r-1}}\right)\right],\right. \\
& 1-\log _{\lambda}\left(1+\frac{\left(\lambda^{1-\left(1-\log _{\lambda}\left(1+\prod_{j=1}^{n}\left(\lambda^{1-m_{A_{j}}}-1\right)^{\omega_{j}}\right)\right)}-1\right)^{r}}{(\lambda-1)^{r-1}}\right), \\
& \left.\left[1-\log _{\lambda}\left(1+\frac{\left(\lambda^{1-\left(1-\log _{\lambda}\left(1+\prod_{j=1}^{n}\left(\lambda^{1-r_{A_{j}}}-1\right)^{\omega_{j}}\right)\right)}-1\right)^{r}}{(\lambda-1)^{r-1}}\right) 1-\log _{\lambda}\left(1+\frac{\left(\lambda^{1-\left(1-\log _{\lambda}\left(1+\prod_{j=1}^{n}\left(\lambda^{1-\tau_{\overline{A_{j}}}}-1\right)^{\omega_{j}}\right)\right)}-1\right)^{r}}{(\lambda-1)^{r-1}}\right)\right]\right) \\
& =\left(\left[1-\log _{\lambda}\left(1+\frac{\prod_{j=1}^{k}\left(\lambda^{1-l_{A_{j}}}-1\right)^{r \omega_{j}}}{(\lambda-1)^{r-1}}\right), 1-\log _{\lambda}\left(1+\frac{\prod_{j=1}^{k}\left(\lambda^{1-\overline{l_{A_{j}}}}-1\right)^{r \omega_{j}}}{(\lambda-1)^{r-1}}\right)\right], 1-\log _{\lambda}\left(1+\frac{\prod_{j=1}^{k}\left(\lambda^{1-m_{A_{j}}}-1\right)^{r \omega_{j}}}{(\lambda-1)^{r-1}}\right)\right. \text {, } \\
& \left.\left[1-\log _{\lambda}\left(1+\frac{\prod_{j=1}^{k}\left(\lambda^{1-r_{A_{j}}}-1\right)^{r \omega_{j}}}{(\lambda-1)^{r-1}}\right), 1-\log _{\lambda}\left(1+\frac{\prod_{j=1}^{k}\left(\lambda^{1-\overline{r_{A_{j}}}}-1\right)^{r \omega_{j}}}{(\lambda-1)^{r-1}}\right)\right]\right),
\end{aligned}
$$

therefore, we have

$$
\begin{array}{r}
\operatorname{TIT}^{2} \text { FFWA }_{\omega}\left(r \cdot{ }_{F} \widetilde{A}_{1}, r \cdot{ }_{F} \widetilde{A}_{2}, \ldots, r{ }_{F} \widetilde{A}_{n}\right) \\
=r \cdot_{F} \text { TIT2FFWA } \\
\left(\widetilde{A}_{1}, \widetilde{A}_{2}, \ldots, \widetilde{A}_{n}\right) .
\end{array}
$$

Thus, the proof is completed.

Theorem 12. Let $\widetilde{A}_{j}=\left(\left[l_{A_{j}}, \overline{l_{A_{j}}}\right], m_{A_{j}},\left[r_{A_{j}}, \overline{r_{A_{j}}}\right]\right)(j=$ $1,2, \ldots, n)$ be a collection of TIT2FNs. If $r>0, \widetilde{A}_{n+1}=$ $\left(\left[{\underline{l_{n+1}}}, \overline{l_{A_{n+1}}}\right], m_{A_{n+1}},\left[\underline{r_{A_{n+1}}}, \overline{r_{A_{n+1}}}\right]\right)$ is a TIT2FN on $X$, then

$$
\begin{aligned}
& \text { TIT2FFWA }\left(r \cdot{ }_{F} \widetilde{A}_{1} \bigoplus_{F} \widetilde{A}_{n+1},\right. \\
& \left.r \quad r{ }_{F} \widetilde{A}_{2} \bigoplus_{F} \widetilde{A}_{n+1}, \ldots, r \cdot{ }_{F} \widetilde{A}_{n} \bigoplus_{F} \widetilde{A}_{n+1}\right) \\
& =r \cdot{ }_{F} T I T 2 F F W A_{\omega}\left(\widetilde{A}_{1}, \widetilde{A}_{2}, \ldots, \widetilde{A}_{n}\right) \bigoplus_{F} \widetilde{A}_{n+1} .
\end{aligned}
$$

Proof. According to Theorems 10 and 11, we can easily prove Theorem 12.

Theorem 13. Let $\widetilde{A}_{j}=\left(\left[l_{A_{j}}, \overline{l_{A_{j}}}\right], m_{A_{j}},\left[r_{A_{j}}, \overline{r_{A_{j}}}\right]\right)(j=$ $1,2, \ldots, n)$ be a collection of TIT2FNs, and assume that $\lambda>1$.
As $\lambda \rightarrow 1$, the TIT2FFAA operator approaches the following limit:

$$
\begin{aligned}
\lim _{\lambda \rightarrow 1} \operatorname{TIT} \mathrm{FFWA}_{\omega}\left(\widetilde{A}_{1}, \widetilde{A}_{2}, \ldots, \widetilde{A}_{n}\right) \\
=\left(\left[1-\prod_{j=1}^{n}\left(1-\underline{l_{A_{j}}}\right)^{\omega_{j}}, 1-\prod_{j=1}^{n}\left(1-\overline{l_{A_{j}}}\right)^{\omega_{j}}\right],\right. \\
1-\prod_{j=1}^{n}\left(1-m_{A_{j}}\right)^{\omega_{j}}, \\
{\left.\left[1-\prod_{j=1}^{n}\left(1-\underline{r_{A_{j}}}\right)^{\omega_{j}}, 1-\prod_{j=1}^{n}\left(1-\overline{r_{A_{j}}}\right)^{\omega_{j}}\right]\right) . }
\end{aligned}
$$

Proof. From Theorem 7, we have

$$
\begin{aligned}
\lim _{\lambda \rightarrow 1} \operatorname{TIT}_{2} \text { FFWA }_{\omega}\left(\widetilde{A}_{1}, \widetilde{A}_{2}, \ldots, \widetilde{A}_{n}\right) \\
=\left(\left[\lim _{\lambda \rightarrow 1}\left(1-\log _{\lambda}\left(1+\prod_{j=1}^{n}\left(\lambda^{1-l_{A_{j}}}-1\right)^{\omega_{j}}\right)\right),\right.\right. \\
\left.\lim _{\lambda \rightarrow 1}\left(1-\log _{\lambda}\left(1+\prod_{j=1}^{n}\left(\lambda^{1-\overline{l_{A_{j}}}}-1\right)^{\omega_{j}}\right)\right)\right], \\
\lim _{\lambda \rightarrow 1}\left(1-\log _{\lambda}\left(1+\prod_{j=1}^{n}\left(\lambda^{1-m_{A_{j}}}-1\right)^{\omega_{j}}\right)\right),
\end{aligned}
$$




$$
\begin{aligned}
& {\left[\lim _{\lambda \rightarrow 1}\left(1-\log _{\lambda}\left(1+\prod_{j=1}^{n}\left(\lambda^{1-r_{A_{j}}}-1\right)^{\omega_{j}}\right)\right),\right.} \\
& \left.\lim _{\lambda \rightarrow 1}\left(1-\log _{\lambda}\left(1+\prod_{j=1}^{n}\left(\lambda^{1-\overline{r_{A_{j}}}}-1\right)^{\omega_{j}}\right)\right)\right] .
\end{aligned}
$$

Since the five reference points have the same mathematical form, so we take the first reference point as an example; that is,

$$
\begin{gathered}
\lim _{\lambda \rightarrow 1}\left(1-\log _{\lambda}\left(1+\prod_{j=1}^{n}\left(\lambda^{1-l_{A_{j}}}-1\right)^{\omega_{j}}\right)\right) \\
=1-\prod_{j=1}^{n}\left(1-\underline{l_{A_{j}}}\right)^{\omega_{j}} .
\end{gathered}
$$

As $\lambda \rightarrow 1$, then $\prod_{j=1}^{n}\left(\lambda^{1-l_{A_{j}}}-1\right)^{\omega_{j}} \rightarrow 0$, by logarithmic transform and the rule of equivalent infinitesimal replacement:

$$
\begin{aligned}
\log _{\lambda}\left(1+\prod_{j=1}^{n}\left(\lambda^{1-l_{A_{j}}}-1\right)^{\omega_{j}}\right) \\
=\frac{\ln \left(1+\prod_{j=1}^{n}\left(\lambda^{1-l_{A_{j}}}-1\right)^{\omega_{j}}\right)}{\ln \lambda} \longrightarrow \frac{\prod_{j=1}^{n}\left(\lambda^{1-l_{A_{j}}}-1\right)^{\omega_{j}}}{\ln \lambda} .
\end{aligned}
$$

Based on Taylor's expansion formula, we have

$$
\lambda^{1-l_{A_{j}}}=1+\left(1-\underline{l_{A_{j}}}\right) \ln \lambda+\frac{\left(1-\underline{l_{A_{j}}}\right)}{2}(\ln \lambda)^{2}+\cdots
$$

Also since $\lambda>1$, then $\ln \lambda>0, \lambda \stackrel{1-l_{A_{j}}}{=}=1+\left(1-\underline{l_{A_{j}}}\right) \ln \lambda+$ $O(\ln \lambda)$, and it follows that

$$
\lambda^{1-l_{A_{j}}}-1 \longrightarrow\left(1-\underline{l_{A_{j}}}\right) \ln \lambda
$$

Therefore,

$$
\begin{aligned}
& \left(\lambda \stackrel{1-l_{A_{j}}}{\underline{\omega_{0}}}-1\right)^{\omega_{j}} \longrightarrow\left(\left(1-\underline{l_{A_{j}}}\right) \ln \lambda\right)^{\omega_{j}} \\
& \Longrightarrow\left(\lambda^{1-l_{A_{j}}}-1\right)^{\omega_{j}} \longrightarrow\left(1-\underline{l_{A_{j}}}\right)^{\omega_{j}}(\ln \lambda)^{\omega_{j}} \\
& \Longrightarrow \prod_{j=1}^{n}\left(\lambda^{1-l_{A_{j}}}-1\right)^{\omega_{j}} \longrightarrow \prod_{j=1}^{n}\left(1-\underline{l_{A_{j}}}\right)^{\omega_{j}} \prod_{j=1}^{n}(\ln \lambda)^{\omega_{j}} \\
& \Longrightarrow \prod_{j=1}^{n}\left(\lambda^{1-l_{A_{j}}}-1\right)^{\omega_{j}} \longrightarrow \prod_{j=1}^{n}\left(1-\underline{l_{A_{j}}}\right)^{\omega_{j}}(\ln \lambda)^{\sum_{j=1}^{n} \omega_{j}}
\end{aligned}
$$

$$
\begin{aligned}
& \Longrightarrow \prod_{j=1}^{n}\left(\lambda^{1-l_{A_{j}}}-1\right)^{\omega_{j}} \longrightarrow \prod_{j=1}^{n}\left(1-\underline{l_{A_{j}}}\right)^{\omega_{j}} \ln \lambda \\
& \Longrightarrow \frac{\prod_{j=1}^{n}\left(\lambda^{1-l_{A_{j}}}-1\right)^{\omega_{j}}}{\ln \lambda} \longrightarrow \prod_{j=1}^{n}\left(1-\underline{l_{A_{j}}}\right)^{\omega_{j}} .
\end{aligned}
$$

Then we have

$$
\begin{aligned}
\lim _{\lambda \rightarrow 1}( & \left.1-\log _{\lambda}\left(1+\prod_{j=1}^{n}\left(\lambda^{1-l_{A_{j}}}-1\right)^{\omega_{j}}\right)\right) \\
= & \lim _{\lambda \rightarrow 1}\left(1-\frac{\ln \left(1+\prod_{j=1}^{n}\left(\lambda^{1-l_{A_{j}}}-1\right)^{\omega_{j}}\right)}{\ln \lambda}\right) \\
= & 1-\lim _{\lambda \rightarrow 1} \frac{\prod_{j=1}^{n}\left(\lambda^{1-l_{A_{j}}}-1\right)^{\omega_{j}}}{\ln \lambda} \\
= & 1-\prod_{j=1}^{n}\left(1-\underline{l_{A_{j}}}\right)^{\omega_{j}} .
\end{aligned}
$$

Similarly, we have

$$
\begin{aligned}
\lim _{\lambda \rightarrow 1}\left(1-\log _{\lambda}\left(1+\prod_{j=1}^{n}\left(\lambda^{1-\overline{l_{A_{j}}}}-1\right)^{\omega_{j}}\right)\right) \\
=1-\prod_{j=1}^{n}\left(1-\overline{l_{A_{j}}}\right)^{\omega_{j}}, \\
\lim _{\lambda \rightarrow 1}\left(1-\log _{\lambda}\left(1+\prod_{j=1}^{n}\left(\lambda^{1-m_{A_{j}}}-1\right)^{\omega_{j}}\right)\right) \\
=1-\prod_{j=1}^{n}\left(1-m_{A_{j}}\right)^{\omega_{j}}, \\
\lim _{\lambda \rightarrow 1}\left(1-\log _{\lambda}\left(1+\prod_{j=1}^{n}\left(\lambda^{1-r_{A_{j}}}-1\right)^{\omega_{j}}\right)\right) \\
\quad=1-\prod_{j=1}^{n}\left(1-\underline{r_{A_{j}}}\right)^{\omega_{j}}, \\
\lim _{\lambda \rightarrow 1}\left(1-\log _{\lambda}\left(1+\prod_{j=1}^{n}\left(\lambda^{1-\overline{r_{A_{j}}}}-1\right)^{\omega_{j}}\right)\right) \\
=1-\prod_{j=1}^{n}\left(1-\overline{r_{A_{j}}}\right)^{\omega_{j}}
\end{aligned}
$$

which completes the proof of Theorem 13.

Theorem 14. Let $\widetilde{A}_{j}=\left(\left[l_{A_{j}}, \overline{l_{A_{j}}}\right], m_{A_{j}},\left[r_{A_{j}}, \overline{r_{A_{j}}}\right]\right)(j=$ $1,2, \ldots, n)$ be a collection of TIT2FNs, and assume that 
$\lambda>1$. As $\lambda \rightarrow+\infty$, the TIT2FFWA operator approaches the following limit:

$$
\begin{aligned}
& \lim _{\lambda \rightarrow \infty} \operatorname{TIT2FFWA}_{\omega}\left(\widetilde{A}_{1}, \widetilde{A}_{2}, \ldots, \widetilde{A}_{n}\right) \\
&=\left(\left[\sum_{j=1}^{n} \omega_{j} l_{A_{j}}, \sum_{j=1}^{n} \omega_{j} \overline{l_{A_{j}}}\right],\right. \\
&\left.\sum_{j=1}^{n} \omega_{j} m_{A_{j}},\left[\sum_{j=1}^{n} \underline{\omega_{j} r_{A_{j}}}, \sum_{j=1}^{n} \omega_{j} \overline{r_{A_{j}}}\right]\right) .
\end{aligned}
$$

Proof. By Theorem 7, we have

$$
\begin{aligned}
\lim _{\lambda \rightarrow 1} \operatorname{TIT} \text { FFWA }_{\omega}\left(\widetilde{A}_{1}, \widetilde{A}_{2}, \ldots, \widetilde{A}_{n}\right) \\
=\left(\left[\lim _{\lambda \rightarrow 1}\left(1-\log _{\lambda}\left(1+\prod_{j=1}^{n}\left(\lambda^{1-l_{A_{j}}}-1\right)^{\omega_{j}}\right)\right),\right.\right. \\
\left.\lim _{\lambda \rightarrow 1}\left(1-\log _{\lambda}\left(1+\prod_{j=1}^{n}\left(\lambda^{1-\overline{l_{A_{j}}}}-1\right)^{\omega_{j}}\right)\right)\right], \\
\lim _{\lambda \rightarrow 1}\left(1-\log _{\lambda}\left(1+\prod_{j=1}^{n}\left(\lambda^{1-m_{A_{j}}}-1\right)^{\omega_{j}}\right)\right), \\
\quad\left[\lim _{\lambda \rightarrow 1}\left(1-\log _{\lambda}\left(1+\prod_{j=1}^{n}\left(\lambda^{1-r_{A_{j}}}-1\right)^{\omega_{j}}\right)\right),\right. \\
\left.\lim _{\lambda \rightarrow 1}\left(1-\log _{\lambda}\left(1+\prod_{j=1}^{n}\left(\lambda^{1-\overline{r_{A_{j}}}}-1\right)^{\omega_{j}}\right)\right)\right] .
\end{aligned}
$$

Since the five reference points have the same mathematical form, so we take the first reference point as an example; that is,

$$
\lim _{\lambda \rightarrow+\infty}\left(1-\log _{\lambda}\left(1+\prod_{j=1}^{n}\left(\lambda^{1-l_{A_{j}}}-1\right)^{\omega_{j}}\right)\right)=\sum_{j=1}^{n} \underline{\omega_{j} l_{A_{j}} .}
$$

As $\lambda \rightarrow+\infty$ by logarithmic transform, we have

$$
\begin{array}{r}
\lim _{\lambda \rightarrow+\infty}\left(1-\log _{\lambda}\left(1+\prod_{j=1}^{n}\left(\lambda^{1-l_{A_{j}}}-1\right)^{\omega_{j}}\right)\right) \\
=1-\lim _{\lambda \rightarrow+\infty} \frac{\ln \left(1+\prod_{j=1}^{n}\left(\lambda^{1-l_{A_{j}}}-1\right)^{\omega_{j}}\right)}{\ln \lambda} .
\end{array}
$$

Based on L'Hospital's rule, it follows that

$$
\begin{aligned}
& \lim _{\lambda \rightarrow+\infty}\left(1-\log _{\lambda}\left(1+\prod_{j=1}^{n}\left(\lambda^{1-l_{A_{j}}}-1\right)^{\omega_{j}}\right)\right) \\
& =1-\lim _{\lambda \rightarrow+\infty} \frac{\ln \left(1+\prod_{j=1}^{n}\left(\lambda^{1-l_{A_{j}}}-1\right)^{\omega_{j}}\right)}{\ln \lambda} \\
& =1-\lim _{\lambda \rightarrow+\infty}\left(\frac{\prod_{j=1}^{n}\left(\lambda^{1-l_{A_{j}}}-1\right)^{\omega_{j}}}{1+\prod_{j=1}^{n}\left(\lambda^{1-l_{A_{j}}}-1\right)^{\omega_{j}}}\right. \\
& \left.\times\left(\sum_{j=1}^{n} \omega_{j}\left(1-\underline{l_{A_{j}}}\right) \frac{\lambda^{-l_{A_{j}}}}{\lambda^{1-l_{A_{j}}}-1}\right)\right) \times\left(\frac{1}{\lambda}\right)^{-1} \\
& =1-\lim _{\lambda \rightarrow+\infty} \frac{\prod_{j=1}^{n}\left(\lambda^{1-l_{A_{j}}}-1\right)^{\omega_{j}}}{1+\prod_{j=1}^{n}\left(\lambda^{1-l_{A_{j}}}-1\right)^{\omega_{j}}} \\
& \times\left(\sum_{j=1}^{n} \omega_{j}\left(1-\underline{l_{A_{j}}}\right) \frac{\lambda^{1-l_{A_{j}}}}{\lambda^{1-\underline{A_{A_{j}}}}-1}\right) \\
& =1-\sum_{j=1}^{n} \omega_{j}\left(1-\underline{l_{A_{j}}}\right) \\
& =\sum_{j=1}^{n} \omega_{j} l_{A_{j}} .
\end{aligned}
$$

Similarly, we have

$$
\begin{aligned}
& \lim _{\lambda \rightarrow \infty}\left(1-\log _{\lambda}\left(1+\prod_{j=1}^{n}\left(\lambda^{1-\overline{l_{A_{j}}}}-1\right)^{\omega_{j}}\right)\right)=\sum_{j=1}^{n} \omega_{j} \overline{l_{A_{j}}} \\
& \lim _{\lambda \rightarrow \infty}\left(1-\log _{\lambda}\left(1+\prod_{j=1}^{n}\left(\lambda^{1-m_{A_{j}}}-1\right)^{\omega_{j}}\right)\right)=\sum_{j=1}^{n} \omega_{j} m_{A_{j}} \\
& \lim _{\lambda \rightarrow \infty}\left(1-\log _{\lambda}\left(1+\prod_{j=1}^{n}\left(\lambda^{1-r_{A_{j}}}-1\right)^{\omega_{j}}\right)\right)=\sum_{j=1}^{n} \omega_{j} r_{A_{j}} \\
& \lim _{\lambda \rightarrow \infty}\left(1-\log _{\lambda}\left(1+\prod_{j=1}^{n}\left(\lambda^{1-\overline{r_{A_{j}}}}-1\right)^{\omega_{j}}\right)\right)=\sum_{j=1}^{n} \omega_{j} \overline{r_{A_{j}}},
\end{aligned}
$$

which completes the proof of Theorem 14.

Theorem 15. For given arguments $\widetilde{A}_{j}(j=1,2, \ldots, n)$, and $\lambda \in(1,+\infty)$, the TIT2FFWA operator is monotonically decreasing with respect to $\lambda$.

Proof. In order to verify that the TIT2FFWA operator is monotonically decreasing with respect to $\lambda$, we should only prove that every reference point function is monotonically 
decreasing with respect to $\lambda$. Due to the space limit, we take the first reference point function as an example.

Let $T_{l_{A}}(\lambda)=1-\log _{\lambda}\left(1+\prod_{j=1}^{n}\left(\lambda^{1-l_{A_{j}}}-1\right)^{\omega_{j}}\right)$, and taking the derivative of $T_{\underline{l_{A}}}(\lambda)$ with respect to $\lambda$, we obtain

$\frac{d T_{l_{A}}(\lambda)}{d \lambda}$

$=-\frac{\prod_{j=1}^{n}\left(\lambda^{1-l_{A_{j}}}-1\right)^{w_{j}}\left(\sum_{j=1}^{n}\left(w_{j}\left(1-\frac{l_{A_{j}}}{\underline{w_{j}}}\right) /\left(\lambda-\lambda^{l_{A_{j}}}\right)\right)\right)}{\left(1+\prod_{j=1}^{n}\left(\lambda^{1-l_{A_{j}}}-1\right)^{w^{\prime}} \lambda\right.}$.

Since $\lambda>1,0 \leq l_{A_{j}} \leq 1$, we can easily prove that

$$
\frac{d T_{l_{A}}(\lambda)}{d \lambda}<0 .
$$

Hence, $T_{l_{A}}(\lambda)$ is monotonically decreasing with respect to $\lambda$, which implies that TIT2FFWA operator is monotonically decreasing with respect to $\lambda$. Thus, the proof is completed.

Theorem 16. Let $\widetilde{A}_{j}=\left(\left[l_{A_{j}}, \overline{l_{A_{j}}}\right], m_{A_{j}},\left[r_{A_{j}}, \overline{r_{A_{j}}}\right]\right)(j=$ $1,2, \ldots, n)$ be a collection of $\overline{T I T} 2 F N s$, for any $\lambda \in(1,+\infty)$; then

$$
\begin{aligned}
& \sup \left\{\operatorname{TIT} 2 F F W A_{\omega}\left(\widetilde{A}_{1}, \widetilde{A}_{2}, \ldots, \widetilde{A}_{n}\right)\right\} \\
& =\left(\left[1-\prod_{j=1}^{n}\left(1-\underline{l_{A_{j}}}\right)^{\omega_{j}}, 1-\prod_{j=1}^{n}\left(1-\overline{l_{A_{j}}}\right)^{\omega_{j}}\right]\right. \text {, } \\
& 1-\prod_{j=1}^{n}\left(1-m_{A_{j}}\right)^{\omega_{j}} \\
& \left.\left[1-\prod_{j=1}^{n}\left(1-\underline{r_{A_{j}}}\right)^{\omega_{j}}, 1-\prod_{j=1}^{n}\left(1-\overline{r_{A_{j}}}\right)^{\omega_{j}}\right]\right), \\
& \inf \left\{\operatorname{TIT} 2 F F W A_{\omega}\left(\widetilde{A}_{1}, \widetilde{A}_{2}, \ldots, \widetilde{A}_{n}\right)\right\} \\
& =\left(\left[\sum_{j=1}^{n} \underline{\omega_{j}} l_{A_{j}}, \sum_{j=1}^{n} \omega_{j} \overline{l_{A_{j}}}\right]\right. \text {, } \\
& \left.\sum_{j=1}^{n} \omega_{j} m_{A_{j}},\left[\sum_{j=1}^{n} \omega_{j} r_{A_{j}}, \sum_{j=1}^{n} \omega_{j} \overline{r_{A_{j}}}\right]\right) \text {. }
\end{aligned}
$$

Proof. Based on Theorems 13-15, the result is obvious.

\subsection{Triangular Interval Type-2 Fuzzy Frank Weighted Geometric Operator}

Definition 17. Let $\widetilde{A}_{j}=\left(\left[l_{A_{j}}, \overline{l_{A_{j}}}\right], m_{A_{j}},\left[r_{A_{j}}, \overline{r_{A_{j}}}\right]\right)(j=$ $1,2, \ldots, n)$ be a collection of TIT2FNs, and let TIT2FFWG: $\Omega^{n} \rightarrow \Omega$; if

$$
\begin{aligned}
\text { TIT2FFWG } & \left(\widetilde{A}_{1}, \widetilde{A}_{2}, \ldots, \widetilde{A}_{n}\right) \\
= & \widetilde{A}_{1}^{{ }_{F} \omega_{1}} \bigotimes_{F} \widetilde{A}_{2}^{{ }^{F}} \omega^{2} \bigotimes_{F} \cdots \bigotimes_{F}{\widetilde{A^{F}}}_{n}{ }^{\omega_{n}},
\end{aligned}
$$

then the function TIT2FFWG is called a triangular interval type-2 fuzzy Frank weighted geometric (TIT2FFWG) operator, where $\omega=\left(\omega_{1}, \omega_{2}, \ldots, \omega_{n}\right)^{T}$ is the weight vector of $\widetilde{A}_{j}(j=1,2, \ldots, n), \omega_{j} \geq 0$, and $\sum_{j=1}^{n} \omega_{j}=1$. In particular, if $\omega=(1 / n, 1 / n, \ldots, 1 / n)^{T}$, then the TIT2FFWG operator is reduced to a triangular interval type-2 fuzzy Frank geometric averaging (TIT2FFGA) operator of dimension $n$, which is defined as follows:

$$
\begin{aligned}
& {\operatorname{TIT} 2 F_{F G}}_{\omega}\left(\widetilde{A}_{1}, \widetilde{A}_{2}, \ldots, \widetilde{A}_{n}\right) \\
& =\left(\widetilde{A}_{1} \bigotimes_{F} \widetilde{A}_{2} \bigotimes_{F} \cdots \bigotimes_{F} \widetilde{A}_{n}\right)^{{ }_{F} 1 / n} .
\end{aligned}
$$

Based on the Frank operation laws from Theorem 5, we can derive Theorem 18 .

Theorem 18. Let $\widetilde{A}_{j}=\left(\left[l_{A_{j}}, \overline{l_{A_{j}}}\right], m_{A_{j}},\left[r_{A_{j}}, \overline{r_{A_{j}}}\right]\right)(j=$ $1,2, \ldots, n)$ be a collection of $\overline{T I T} 2 F N$; then their aggregated value by TIT2FFWG operator is still a TIT2FN, and

$$
\begin{aligned}
& \operatorname{TIT2FFWG}\left(\widetilde{A}_{1}, \widetilde{A}_{2}, \ldots, \widetilde{A}_{n}\right) \\
& =\left(\left[\log _{\lambda}\left(1+\prod_{j=1}^{n}\left(\lambda^{l_{A_{j}}}-1\right)^{\omega_{j}}\right)\right.\right. \text {, } \\
& \left.\log _{\lambda}\left(1+\prod_{j=1}^{n}\left(\lambda^{\overline{l_{A_{j}}}}-1\right)^{\omega_{j}}\right)\right], \\
& \log _{\lambda}\left(1+\prod_{j=1}^{n}\left(\lambda^{m_{A_{j}}}-1\right)^{\omega_{j}}\right), \\
& {\left[\log _{\lambda}\left(1+\prod_{j=1}^{n}\left(\lambda \stackrel{r_{A_{j}}}{ }-1\right)^{\omega_{j}}\right),\right.} \\
& \left.\log _{\lambda}\left(1+\prod_{j=1}^{n}\left(\lambda^{\overline{r_{A_{j}}}}-1\right)^{\omega_{j}}\right)\right],
\end{aligned}
$$

where $\omega=\left(\omega_{1}, \omega_{2}, \ldots, \omega_{n}\right)^{T}$ is the weight vector of $\widetilde{A}_{j}(j=$ $1,2, \ldots, n), \omega_{j}>0$, and $\sum_{j=1}^{n} \omega_{j}=1$.

Proof. The proof of Theorem 18 is similar to Theorem 7. 
Theorem 19 (idempotency). Let $\widetilde{A}_{j}=\left(\left[l_{A_{j}}, \overline{l_{A_{j}}}\right], m_{A_{j}}\right.$, $\left.\left[r_{A_{j}}, \overline{r_{A_{j}}}\right]\right)(j=1,2, \ldots, n)$ be a collection of TIT2FNs, where $\overline{0 \leq} l_{A_{j}} \leq \overline{l_{A_{j}}} \leq m_{A_{j}} \leq r_{A_{j}} \leq \overline{r_{A_{j}}} \leq 1$. If all $\widetilde{A}_{j}(j=1,2, \ldots, n)$ are equal, that is, $\widetilde{A}_{j}=\overline{\widetilde{A}}$, for all $j$, then

$$
\operatorname{TIT}_{2 F F W G}\left(\widetilde{A}_{1}, \widetilde{A}_{2}, \ldots, \widetilde{A}_{n}\right)=\widetilde{A} .
$$

Proof. The proof of Theorem 19 is similar to Theorem 8.

Theorem 20 (boundary). Let $\widetilde{A}_{j}=\left(\left[l_{A_{j}}, \overline{l_{A_{j}}}\right], m_{A_{j}}\right.$, $\left.\left[r_{A_{j}}, \overline{r_{A_{j}}}\right]\right)(j=1,2, \ldots, n)$ be a collection of $\overline{\text { TIT } 2 F N s, ~ w h e r e ~}$ $\overline{0 \leq} l_{A_{j}} \leq \overline{l_{A_{j}}} \leq m_{A_{j}} \leq \underline{r_{A_{j}}} \leq \overline{r_{A_{j}}} \leq 1$, and let

$$
\begin{gathered}
\widetilde{A}^{+}=\left(\left[\max _{j=1}^{n}\left\{\underline{\left.l_{A_{j}}\right\}}, \max _{j=1}^{n}\left\{\overline{l_{A_{j}}}\right\}\right], \max _{j=1}^{n}\left\{m_{A_{j}}\right\},\right.\right. \\
{\left[\max _{j=1}^{n}\left\{\underline{\left.r_{A_{j}}\right\}}, \max _{j=1}^{n}\left\{\overline{r_{A_{j}}}\right\}\right]\right),} \\
\widetilde{A}^{-}=\left(\left[\min _{j=1}^{n}\left\{\underline{l_{A_{j}}}\right\}, \min _{j=1}^{n}\left\{\overline{l_{A_{j}}}\right\}\right], \min _{j=1}^{n}\left\{m_{A_{j}}\right\},\right. \\
\left.\left[\min _{j=1}^{n}\left\{\underline{r_{A_{j}}}\right\}, \min _{j=1}^{n}\left\{\overline{r_{A_{j}}}\right\}\right]\right) .
\end{gathered}
$$

Then

$$
\widetilde{A}^{-} \leq \operatorname{TIT2FFWG}\left(\widetilde{A}_{1}, \widetilde{A}_{2}, \ldots, \widetilde{A}_{n}\right) \leq \widetilde{A}^{+} .
$$

Proof. The proof of Theorem 20 is similar to Theorem 9.

Theorem 21. Let $\widetilde{A}_{j}=\left(\left[l_{A_{j}}, \overline{l_{A_{j}}}\right], m_{A_{j}},\left[r_{A_{j}}, \overline{r_{A_{j}}}\right]\right)(j=$ $1,2, \ldots, n)$ be a collection of $\overline{T I T 2 F N s, ~ w h e r e ~} 0 \leq l_{A_{j}} \leq$ $\overline{l_{A_{j}}} \leq m_{A_{j}} \leq \underline{r_{A_{j}}} \leq \overline{r_{A_{j}}} \leq$ 1. If $\widetilde{A_{n+1}}=$ $\left(\left[l_{A_{n+1}}, \overline{l_{A_{n+1}}}\right], m_{A_{n+1}},\left[\overline{r_{A_{n+1}}}, \overline{r_{A_{n+1}}}\right]\right)$ is a triangular interval type$2 \overline{\text { fuzzy number on } X \text {, then }}$

$$
\begin{aligned}
& \operatorname{TIT2FFWG}_{\omega}\left(\widetilde{A}_{1} \bigoplus_{F} \widetilde{A}_{n+1}, \widetilde{A}_{2} \bigoplus_{F} \widetilde{A}_{n+1}, \ldots, \widetilde{A}_{n} \bigoplus_{F} \widetilde{A}_{n+1}\right) \\
& =\operatorname{TIT2FFWG} G_{\omega}\left(\widetilde{A}_{1}, \widetilde{A}_{2}, \ldots, \widetilde{A}_{n}\right) \bigoplus_{F} \widetilde{A}_{n+1} .
\end{aligned}
$$

Proof. The proof of Theorem 21 is similar to Theorem 10.

Theorem 22. Let $\widetilde{A}_{j}=\left(\left[l_{A_{j}}, \overline{l_{A_{j}}}\right], m_{A_{j}},\left[r_{A_{j}}, \overline{r_{A_{j}}}\right]\right)(j=$ $1,2, \ldots, n)$ be a collection of TIT2FNs, where $0 \leq l_{A_{j}} \leq \overline{l_{A_{j}}} \leq$ $m_{A_{j}} \leq r_{A_{j}} \leq \overline{r_{A_{j}}} \leq 1$. If $r>0$, then

$$
\begin{aligned}
& \text { TIT2FFWG }\left(\widetilde{A}_{1}^{F^{r}}, \widetilde{A}_{2}^{{ }^{r}}{ }^{r}, \ldots, \widetilde{A}_{n}^{F^{r}}\right) \\
& =\left(\operatorname{TIT} 2 F F W G_{\omega}\left(\widetilde{A}_{1}, \widetilde{A}_{2}, \ldots, \widetilde{A}_{n}\right)\right)^{{ }_{F}{ }^{r}} .
\end{aligned}
$$

Proof. The proof of Theorem 22 is similar to Theorem 11.

Theorem 23. Let $\widetilde{A}_{j}=\left(\left[l_{A_{j}}, \overline{l_{A_{j}}}\right], m_{A_{j}},\left[r_{A_{j}}, \overline{r_{A_{j}}}\right]\right)(j=$ $1,2, \ldots, n)$ be a collection of TIT2FNs. If $r>0, \widetilde{A}_{n+1}=$ $\left(\left[\underline{l_{A_{n+1}}}, \overline{l_{A_{n+1}}}\right], m_{A_{n+1}},\left[\underline{r_{A_{n+1}}}, \overline{r_{A_{n+1}}}\right]\right)$ is a TIT2FN on $X$, then

$$
\begin{gathered}
\text { TIT2FFWG }\left(\widetilde{A}_{1}^{{ }^{F}} \bigotimes_{F} \widetilde{A}_{n+1}, \widetilde{A}_{2}^{{ }^{F}} \bigotimes_{F} \widetilde{A}_{n+1}, \ldots, \widetilde{A}_{n}^{{ }^{F}} \bigotimes_{F} \widetilde{A}_{n+1}\right) \\
=\left(\operatorname{TIT} 2 F F W G_{\omega}\left(\widetilde{A}_{1}, \widetilde{A}_{2}, \ldots, \widetilde{A}_{n}\right)\right)^{{ }^{*} r} \bigotimes_{F} \widetilde{A}_{n+1} .
\end{gathered}
$$

Proof. The proof of Theorem 23 is similar to Theorem 12.

Theorem 24. Let $\widetilde{A}_{j}=\left(\left[l_{A_{j}}, \overline{l_{A_{j}}}\right], m_{A_{j}},\left[r_{A_{j}}, \overline{r_{A_{j}}}\right]\right)(j=$ $1,2, \ldots, n)$ be a collection of $T I \overline{T 2} \mathrm{FNs}$, and assume that $\lambda>1$. As $\lambda \rightarrow 1$, the TIT2FFWG operator approaches the following limit:

$$
\begin{aligned}
\lim _{\lambda \rightarrow 1} \operatorname{TIT} 2 F F W G_{\omega}\left(\widetilde{A}_{1}, \widetilde{A}_{2}, \ldots, \widetilde{A}_{n}\right) \\
=\left(\left[\prod_{j=1}^{n}\left(\underline{l_{A_{j}}}\right)^{\omega_{j}}, \prod_{j=1}^{n}\left(\overline{l_{A_{j}}}\right)^{\omega_{j}}\right],\right. \\
\\
\left.\prod_{j=1}^{n}\left(m_{A_{j}}\right)^{\omega_{j}},\left[\prod_{j=1}^{n}\left(\underline{r_{A_{j}}}\right)^{\omega_{j}}, \prod_{j=1}^{n}\left(\overline{r_{A_{j}}}\right)^{\omega_{j}}\right]\right) .
\end{aligned}
$$

Proof. The proof of Theorem 24 is similar to Theorem 13.

Theorem 25. Let $\widetilde{A}_{j}=\left(\left[l_{A_{j}}, \overline{l_{A_{j}}}\right], m_{A_{j}},\left[r_{A_{j}}, \overline{r_{A_{j}}}\right]\right)(j=$ $1,2, \ldots, n)$ be a collection of $\overline{T T T} 2 F N s$, and assume that $\lambda>$ 1. As $\lambda \rightarrow+\infty$, the TIT2FFWG operator approaches the following limit:

$$
\begin{aligned}
\lim _{\lambda \rightarrow \infty} & \operatorname{TIT} 2 F F W G_{\omega}\left(\widetilde{A}_{1}, \widetilde{A}_{2}, \ldots, \widetilde{A}_{n}\right) \\
= & \left(\left[\sum_{j=1}^{n} \omega_{j} l_{A_{j}}, \sum_{j=1}^{n} \omega_{j} \overline{\bar{l}_{A_{j}}}\right],\right. \\
& \left.\sum_{j=1}^{n} \omega_{j} m_{A_{j}},\left[\sum_{j=1}^{n} \underline{\omega_{j} r_{A_{j}}}, \sum_{j=1}^{n} \omega_{j} \overline{r_{A_{j}}}\right]\right) .
\end{aligned}
$$

Proof. The proof of Theorem 25 is similar to Theorem 14.

Theorem 26. For given arguments $\widetilde{A}_{j}(j=1,2, \ldots, n)$, and $\lambda \in(1,+\infty)$, the TIT2FFWG operator is monotonically increasing with respect to $\lambda$.

Proof. The proof of Theorem 26 is similar to Theorem 15. 
Theorem 27. Let $\widetilde{A}_{j}=\left(\left[l_{A_{j}}, \overline{l_{A_{j}}}\right], m_{A_{j}},\left[r_{A_{j}}, \overline{r_{A_{j}}}\right]\right)(j=$ $1,2, \ldots, n)$ be a collection of TIT2FNs, for any $\bar{\lambda} \in(1,+\infty)$; then

$$
\begin{aligned}
& \sup \left\{\operatorname{TIT2FFWG} G_{\omega}\left(\widetilde{A}_{1}, \widetilde{A}_{2}, \ldots, \widetilde{A}_{n}\right)\right\} \\
& =\left(\left[\sum_{j=1}^{n} \omega_{j} l_{A_{j}}, \sum_{j=1}^{n} \omega_{j} \overline{l_{A_{j}}}\right], \sum_{j=1}^{n} \omega_{j} m_{A_{j}},\right. \\
& \left.\left[\sum_{j=1}^{n} \omega_{j} r_{A_{j}}, \sum_{j=1}^{n} \omega_{j} \overline{r_{A_{j}}}\right]\right), \\
& \inf \left\{\operatorname{TIT2FFWG} G_{\omega}\left(\widetilde{A}_{1}, \widetilde{A}_{2}, \ldots, \widetilde{A}_{n}\right)\right\} \\
& =\left(\left[\prod_{j=1}^{n} l_{A_{j}}{ }^{\omega_{j}}, \prod_{j=1}^{n} \bar{l}_{A_{j}} \omega_{j}\right], \prod_{j=1}^{n} m_{A_{j}}^{\omega_{j}},\right. \\
& \left.\left[\prod_{j=1}^{n} l_{A_{j}}{ }^{\omega_{j}}, \prod_{j=1}^{n} \overline{l_{A_{j}} \omega_{j}}\right]\right) .
\end{aligned}
$$

Proof. The proof of Theorem 27 is similar to Theorem 16.

\subsection{The Relationship between the TIT2FFWA Operator and TIT2FFWG Operator}

Theorem 28. Let $\widetilde{A}_{j}=\left(\left[l_{A_{j}}, \overline{l_{A_{j}}}\right], m_{A_{j}},\left[r_{A_{j}}, \overline{r_{A_{j}}}\right]\right)(j=$ $1,2, \ldots, n)$ be a collection of TITTFNs, and $\lambda \overline{\epsilon(1},+\infty)$; then

$$
\begin{aligned}
& \operatorname{TIT2FFWA}_{\omega}\left(\widetilde{A}_{1}, \widetilde{A}_{2}, \ldots, \widetilde{A}_{n}\right) \\
& \quad \geq \operatorname{TIT2FFWG}\left(\widetilde{A}_{1}, \widetilde{A}_{2}, \ldots, \widetilde{A}_{n}\right) .
\end{aligned}
$$

Proof. Based on Theorem 12, we have

$$
\begin{gathered}
\inf \left\{\operatorname{TIT} \operatorname{FFFWA}_{\omega}\left(\widetilde{A}_{1}, \widetilde{A}_{2}, \ldots, \widetilde{A}_{n}\right)\right\} \\
=\left(\left[\sum_{j=1}^{n} \omega_{j} l_{A_{j}}, \sum_{j=1}^{n} \omega_{j} \overline{l_{A_{j}}}\right], \sum_{j=1}^{n} \omega_{j} m_{A_{j}},\right. \\
\left.\left[\sum_{j=1}^{n} \omega_{j} r_{A_{j}}, \sum_{j=1}^{n} \omega_{j} \overline{r_{A_{j}}}\right]\right) .
\end{gathered}
$$

Then it follows that

$$
\begin{gathered}
{\operatorname{TIT} 2 F^{\prime}}_{\geq}\left(\left[\sum_{j=1}^{n} \omega_{j} l_{A_{j}}, \sum_{j=1}^{n} \omega_{j} \overline{l_{A_{j}}}\right], \widetilde{A}_{2}, \ldots, \widetilde{A}_{n}\right) \\
{\left[\sum_{j=1}^{n} m_{A_{j}},\right.} \\
\left.\left[\omega_{j} r_{A_{j}}, \sum_{j=1}^{n} \omega_{j} \overline{r_{A_{j}}}\right]\right) .
\end{gathered}
$$

From Theorem 27, we have

$$
\begin{gathered}
\sup \left\{\operatorname{TIT} 2 \mathrm{FFWG}_{\omega}\left(\widetilde{A}_{1}, \widetilde{A}_{2}, \ldots,{\widetilde{A_{n}}}_{n}\right)\right\} \\
=\left(\left[\sum_{j=1}^{n} \omega_{j} l_{A_{j}}, \sum_{j=1}^{n} \omega_{j} \overline{l_{A_{j}}}\right], \sum_{j=1}^{n} \omega_{j} m_{A_{j}},\right. \\
\left.\left[\sum_{j=1}^{n} \omega_{j} r_{A_{j}}, \sum_{j=1}^{n} \omega_{j} \overline{r_{A_{j}}}\right]\right) .
\end{gathered}
$$

Then

$$
\begin{gathered}
\text { TIT2FFWG }{ }_{\omega}\left(\widetilde{A}_{1}, \widetilde{A}_{2}, \ldots, \widetilde{A}_{n}\right) \\
\leq\left(\left[\sum_{j=1}^{n} \omega_{j} l_{A_{j}}, \sum_{j=1}^{n} \omega_{j} \overline{l_{A_{j}}}\right], \sum_{j=1}^{n} \omega_{j} m_{A_{j}},\right. \\
\left.\left[\sum_{j=1}^{n} \omega_{j} r_{A_{j}}, \sum_{j=1}^{n} \omega_{j} \overline{r_{A_{j}}}\right]\right) .
\end{gathered}
$$

Therefore, according to the transfer of inequality, we can easily deduce that

$$
\begin{aligned}
& \operatorname{TIT2FFWA}_{\omega}\left(\widetilde{A}_{1}, \widetilde{A}_{2}, \ldots, \widetilde{A}_{n}\right) \\
& \quad \geq \operatorname{TIT}_{2} \text { FFWG }_{\omega}\left(\widetilde{A}_{1}, \widetilde{A}_{2}, \ldots, \widetilde{A}_{n}\right) .
\end{aligned}
$$

The proof is completed.

Theorem 28 shows that the values obtained by the TIT2FFWG operator are not bigger than the ones obtained by the TIT2FFWA operator for any $\lambda>1$.

\section{An Approach to Multiple Attribute Decision Making under the Triangular Interval Type-2 Fuzzy Environment}

In this section, we investigate the group decision-making problems under the triangular interval type-2 fuzzy environment based on the proposed Frank aggregation operators in Section 4. First, we describe the MAGDM problems with triangular interval type-2 fuzzy information in this paper.

Let $A=\left(A_{1}, A_{2}, \ldots, A_{m}\right)$ be the set of alternatives, let $D=\left(D_{1}, D_{2}, \ldots, D_{p}\right)$ be the set of decision makers (DMs), and let $e=\left(e_{1}, e_{2}, \ldots, e_{p}\right)$ be the weight vector of DMs, where $e_{k} \geq 0, k=1,2 \ldots, p$, and $\sum_{j=1}^{p} e_{k}=1$. Let $C=\left(C_{1}, C_{2}, \ldots, C_{n}\right)$ be a set of attributes, and let $\omega=$ $\left(\omega_{1}, \omega_{2} \ldots, \omega_{n}\right)$ be a set of weight vectors of them, satisfying $\omega_{j} \in[0,1]$ and $\sum_{j=1}^{n} \omega_{j}=1$. Let $\widetilde{A}^{(k)}=\left(\widetilde{a}_{i j}^{(k)}\right)_{m \times n}$ be a triangular interval type-2 fuzzy decision matrix, where $\widetilde{a}_{i j}^{(k)}$ is measured by TIT2FNs, which is provided by the decision maker $D_{k}$ for the alternative $A_{i}$ with respect to attribute $C_{j}$.

In the following, we will use the TIT2FFWA (or TIT2FFWG) operator to develop an approach to MAGDM with triangular interval type-2 fuzzy information, which involves the following steps. 
Step 1. Normalize the decision-making information matrix $\widetilde{A}^{(k)}=\left(\widetilde{a}_{i j}^{(k)}\right)_{m \times n}$.

In general, attributes are divided into two types; one is benefit attribute (the bigger the better) and the other is cost attribute (the smaller the better); in order to maintain the consistency of the attribute values, we should transform the decision matrix $\widetilde{A}^{(k)}=\left(\widetilde{a}_{i j}^{(k)}\right)_{m \times n}$ into the normalization matrix $\widetilde{R}^{(k)}=\left(\widetilde{r}_{i j}^{(k)}\right)_{m \times n}$ unless all the attributes are the same type. In this paper, we use the following formula to obtain the normalized decision matrix $\widetilde{R}^{(k)}=\left(\widetilde{r}_{i j}^{(k)}\right)_{m \times n}$ :

$$
\widetilde{r}_{i j}^{(k)}= \begin{cases}\widetilde{a}_{i j}^{(k)} & \text { for benefit attribute } C_{j}, \\ \left(\widetilde{a}_{i j}^{(k)}\right)^{c} & \text { for cost attribute } C_{j},\end{cases}
$$

where $\left(\widetilde{a}_{i j}^{(k)}\right)^{c}$ is the complement of $\tilde{a}_{i j}^{(k)}$.

Step 2. Utilize the TIT2FFWA operator

$$
\begin{gathered}
\widetilde{r}_{i j}=\operatorname{TIT2FFWA}\left(\widetilde{r}_{i j}^{(1)}, \widetilde{r}_{i j}^{(2)}, \ldots, \widetilde{r}_{i j}^{(p)}\right) \\
i=1,2, \ldots, m, \quad j=1,2, \ldots, n,
\end{gathered}
$$

or the TIT2FFWG operator

$$
\begin{array}{r}
\widetilde{r}_{i j}=\operatorname{TIT2FFWG}\left(\widetilde{r}_{i j}^{(1)}, \widetilde{r}_{i j}^{(2)}, \ldots, \widetilde{r}_{i j}^{(p)}\right) \\
i=1,2, \ldots, m, \quad j=1,2, \ldots, n
\end{array}
$$

to aggregate all the individual triangular interval type-2 fuzzy decision matrix $\widetilde{R}^{(k)}=\left(\widetilde{r}_{i j}^{(k)}\right)_{m \times n}$ into the collective triangular interval type-2 fuzzy decision matrix $\widetilde{R}=\left(\widetilde{r}_{i j}\right)_{m \times n}$.

Step 3. Aggregate the triangular interval type-2 fuzzy values $\tilde{r}_{i j}$ for each alternative $A_{i}(i=1,2, \ldots, m)$ by using the TIT2FFWA (or TIT2FFWG) operator:

$$
\tilde{r}_{i}=\operatorname{TIT} 2 \mathrm{FFWA}\left(\widetilde{r}_{i 1}, \widetilde{r}_{i 2}, \ldots, \widetilde{r}_{i n}\right) \quad i=1,2, \ldots, m
$$

or

$$
\widetilde{r}_{i}=\operatorname{TIT} 2 \operatorname{FFWG}\left(\widetilde{r}_{i 1}, \widetilde{r}_{i 2}, \ldots, \widetilde{r}_{i n}\right) \quad i=1,2, \ldots, m .
$$

Step 4. Calculate the score values $S\left(\widetilde{r}_{i}\right)(i=1,2, \ldots, m)$ of the overall preference values $\widetilde{r}_{i}(i=1,2, \ldots, m)$.

Step 5. Rank all the alternatives $A_{i}(i=1,2, \ldots, m)$ and select the best one(s) in accordance with $S\left(\widetilde{r}_{i}\right)$. The greater the score values $S\left(\widetilde{r}_{i}\right)$ are, the better the alternatives $A_{i}(i=1,2, \ldots, m)$ will be.

Step 6. End.

\section{Illustrative Example}

In this section, an illustrative example for MAGDM is provided as a demonstration of the application based on the proposed triangular interval type-2 fuzzy Frank aggregation operator in real-world practice situations.
Let us consider a high-tech company, which aims to select a supplier of USB connectors (adapted from [42]). There are four potential suppliers $A_{i}(i=1,2,3,4)$ that have been designated for further evaluation, and assume that there are four attributes to be considered in the evaluation process: (1) $\mathrm{C}_{1}$ : financial; (2) $\mathrm{C}_{2}$ : performance; (3) $\mathrm{C}_{3}$ : technical capacity; and (4) $\mathrm{C}_{4}$ : organizational culture and strategy, and $\omega=(0.35,0.25,0.25,0.15)^{T}$ is the weight vector of them. The decision committee contains three decision makers $D_{k}(k=1,2,3)$ including engineering expert, financial expert, and quality control expert, whose weight vector is $e=(0.35,0.35,0.3)^{T}$. The decision makers $D_{k}(k=1,2,3)$ express the attribute values of the potential supplier $A_{i}(i=$ $1,2,3,4)$ with respect to $C_{i}(i=1,2,3,4)$ by TIT2FNs, respectively, which are listed in Tables 1,2 , and 3 .

Based on the TIT2FFWA operator, the decision-making steps are shown as follows.

Step 1. Consider that all the attributes are benefit type; therefore, the decision matrices do not need normalization; therefore, $\widetilde{R}^{(k)}=\widetilde{A}^{(k)}=\left(\widetilde{a}_{i j}^{(k)}\right)_{4 \times 4}=\left(\widetilde{r}_{i j}^{(k)}\right)_{4 \times 4}$.

Step 2. Utilize the TIT2FFWA operator to aggregate all the individual triangular interval type-2 fuzzy decision matrix $\widetilde{R}^{(k)}=\left(\widetilde{r}_{i j}^{(k)}\right)_{4 \times 4}$ into the collective triangular interval type-2 fuzzy decision matrix $\widetilde{R}=\left(\widetilde{r}_{i j}\right)_{4 \times 4}$, and without the loss of generality, we let $\lambda=2$; the result is shown in Table 4 .

Step 3. Utilize the TIT2FFWA operator to aggregate all the preference values $\widetilde{r}_{i j}(j=1,2,3,4)$ in the $i$ th line of $\widetilde{R}$, and then we can get the overall preference values $\widetilde{r}_{i}(i=1,2,3,4)$ as follows:

$$
\begin{aligned}
& \widetilde{r}_{1}=([0.4738,0.5751], 0.6772,[0.7819,1]), \\
& \widetilde{r}_{2}=([0.5086,0.6109], 0.7146,[0.8224,1]), \\
& \widetilde{r}_{3}=([0.3020,0.4054], 0.5108,[0.6210,1]), \\
& \widetilde{r}_{4}=([0.2872,0.3901], 0.4950,[0.6048,1]) .
\end{aligned}
$$

Step 4. Calculate the score values $S\left(\widetilde{r}_{i}\right)(i=1,2,3,4)$ of the overall preference values $\widetilde{r}_{i}(i=1,2,3,4)$, respectively:

$$
\begin{array}{ll}
S\left(\widetilde{r}_{1}\right)=1.2027, & S\left(\widetilde{r}_{2}\right)=1.2719, \\
S\left(\widetilde{r}_{3}\right)=0.9022, & S\left(\widetilde{r}_{4}\right)=0.8821 .
\end{array}
$$

Step 5. Rank all the alternatives $A_{i}(i=1,2,3,4)$ and select the best one(s) in accordance with $S\left(\widetilde{r}_{i}\right)$.

Since

$$
S\left(\widetilde{r}_{2}\right)>S\left(\widetilde{r}_{1}\right)>S\left(\widetilde{r}_{3}\right)>S\left(\widetilde{r}_{4}\right),
$$

therefore, we have

$$
A_{2}>A_{1}>A_{3}>A_{4}
$$

where the symbol " $>$ " means "superior to." Thus, the best supplier is $A_{2}$. 
TABLE 1: Triangular interval type-2 fuzzy decision matrix $\widetilde{R}^{(1)}$.

\begin{tabular}{lcccc}
\hline & $C_{1}$ & $C_{2}$ & $C_{3}$ & $C_{4}$ \\
\hline$A_{1}$ & $([0.4,0.5], 0.6,[0.7,0.8])$ & $([0.5,0.6], 0.7,[0.8,0.9])$ & $([0.6,0.7], 0.8,[0.9,1])$ & $([0.6,0.7], 0.8,[0.9,1])$ \\
$A_{2}$ & $([0.5,0.6], 0.7,[0.8,0.9])$ & $([0.5,0.6], 0.7,[0.8,0.9])$ & $([0.4,0.5], 0.6,[0.7,0.8])$ & $([0.6,0.7], 0.8,[0.9,1])$ \\
$A_{3}$ & $([0.3,0.4], 0.5,[0.6,0.7])$ & $([0.4,0.5], 0.6,[0.7,0.8])$ & $([0.2,0.3], 0.4,[0.5,0.6])$ & $([0.3,0.4], 0.5,[0.6,0.7])$ \\
$A_{4}$ & $([0.2,0.3], 0.4,[0.5,0.6])$ & $([0.6,0.7], 0.8,[0.9,1])$ & $([0.3,0.4], 0.5,[0.6,0.7])$ & $([0.4,0.5], 0.6,[0.7,0.8])$ \\
\hline
\end{tabular}

TABLE 2: Triangular interval type-2 fuzzy decision matrix $\widetilde{R}^{(2)}$.

\begin{tabular}{ccccc}
\hline & $C_{1}$ & $C_{2}$ & $C_{3}$ & $C_{4}$ \\
\hline$A_{1}$ & $([0.5,0.6], 0.7,[0.8,0.9])$ & $([0.4,0.5], 0.6,[0.7,0.8])$ & $([0.5,0.6], 0.7,[0.8,0.9])$ & $([0.4,0.5], 0.6,[0.7,0.8])$ \\
$A_{2}$ & $([0.6,0.7], 0.8,[0.9,1])$ & $([0.6,0.7], 0.8,[0.9,1])$ & $([0.5,0.6], 0.7,[0.8,0.9])$ & $([0.5,0.6], 0.7,[0.8,0.9])$ \\
$A_{3}$ & $([0.2,0.3], 0.4,[0.5,0.6])$ & $([0.5,0.6], 0.7,[0.8,0.9])$ & $([0.1,0.2], 0.3,[0.4,0.5])$ & $([0.2,0.3], 0.4,[0.5,0.6])$ \\
$A_{4}$ & $([0.3,0.4], 0.5,[0.6,0.7])$ & $([0.4,0.5], 0.6,[0.7,0.8])$ & $([0.2,0.3], 0.4,[0.5,0.6])$ & $([0.1,0.2], 0.3,[0.4,0.5])$ \\
\hline
\end{tabular}

TABLE 3: Triangular interval type-2 fuzzy decision matrix $\widetilde{R}^{(3)}$.

\begin{tabular}{ccccc}
\hline & $C_{1}$ & $C_{2}$ & $C_{3}$ & $C_{4}$ \\
\hline$A_{1}$ & $([0.4,0.5], 0.6,[0.7,0.8])$ & $([0.5,0.6], 0.7,[0.8,0.9])$ & $([0.4,0.5], 0.6,[0.7,0.8])$ & $([0.5,0.6], 0.7,[0.8,0.9])$ \\
$A_{2}$ & $([0.3,0.4], 0.5,[0.6,0.7])$ & $([0.6,0.7], 0.8,[0.9,1])$ & $([0.4,0.5], 0.6,[0.7,0.8])$ & $([0.6,0.7], 0.8,[0.9,1])$ \\
$A_{3}$ & $([0.1,0.2], 0.3,[0.4,0.5])$ & $([0.6,0.7], 0.8,[0.9,1])$ & $([0.3,0.4], 0.5,[0.6,0.7])$ & $([0.4,0.5], 0.6,[0.7,0.8])$ \\
$A_{4}$ & $([0.2,0.3], 0.4,[0.5,0.6])$ & $([0.2,0.3], 0.4,[0.5,0.6])$ & $([0.1,0.2], 0.3,[0.4,0.5])$ & $([0.3,0.4], 0.5,[0.6,0.7])$ \\
\hline
\end{tabular}

TABLE 4: Triangular interval type-2 fuzzy decision matrix $\widetilde{R}$.

\begin{tabular}{ccccc}
\hline & $C_{1}$ & $C_{2}$ & $C_{3}$ & $C_{4}$ \\
\hline \multirow{2}{*}{$A_{1}$} & $([0.4367,0.5372], 0.6379$, & $([0.4667,0.5671], 0.6679$, & $([0.5105,0.6122], 0.7152$, & $([0.5059,0.6078], 0.7109$, \\
& $[0.7393,0.8427])$ & $[0.7692,0.8722])$ & $[0.8217,1])$ & $[0.8180,1])$ \\
$A_{2}$ & $([0.4862,0.5893], 0.6944$, & $([0.5671,0.6679], 0.7692$, & $([0.4367,0.5372], 0.6379$, & $([0.5671,0.6679], 0.7692$, \\
& $[0.8047,1])$ & $[0.8722,1])$ & $[0.7393,0.8427])$ & $[0.8722,1])$ \\
$A_{3}$ & $([0.2081,0.3086], 0.4094$, & $([0.5005,0.6022], 0.7051$, & $([0.1980,0.2986], 0.3994$, & $([0.2986,0.3994], 0.5005$, \\
& $[0.5105,0.6122])$ & $[0.8116,1])$ & $[0.5005,0.6022])$ & $[0.6022,0.7051])$ \\
$A_{4}$ & $([0.2362,0.3364], 0.4367$, & $([0.4283,0.5333], 0.6413$, & $([0.2081,0.3086], 0.4094$, & $([0.2733,0.3750], 0.4773$, \\
& $[0.5372,0.6379])$ & $[0.7577,1])$ & $[0.5105,0.6122])$ & $[0.5807,0.6862])$ \\
\hline
\end{tabular}

TABLE 5: Triangular interval type-2 fuzzy decision matrix $\widetilde{R}$.

\begin{tabular}{ccccc}
\hline & $C_{1}$ & $C_{2}$ & $C_{3}$ & $C_{4}$ \\
\hline \multirow{2}{*}{$A_{1}$} & $([0.4329,0.5333], 0.6336$, & $([0.4628,0.5633], 0.6636$, & $([0.4995,0.6006], 0.7014$, & $([0.4941,0.5952], 0.6961$, \\
& $[0.7338,0.8340])$ & $[0.7638,0.8640])$ & $[0.8020,0.9024])$ & $[0.7967,0.8972])$ \\
$A_{2}$ & $([0.4599,0.5633], 0.6656$, & $([0.5633,0.6636], 0.7638$, & $([0.4329,0.5333], 0.6336$, & $([0.5633,0.6636], 0.7638$, \\
& $[0.7672,0.8684])$ & $[0.8640,0.9642])$ & $[0.7338,0.8340])$ & $[0.8640,0.9642])$ \\
$A_{3}$ & $([0.1884,0.2949], 0.3978$, & $([0.4895,0.5906], 0.6914$, & $([0.1783,0.2848], 0.3878$, & $([0.2848,0.3878], 0.4895$, \\
& $[0.4995,0.6006])$ & $[0.7919,0.8924])$ & $[0.4895,0.5906])$ & $[0.5906,0.6914])$ \\
$A_{4}$ & $([0.2308,0.3321], 0.4329$, & $([0.3789,0.4869], 0.5918$, & $([0.1884,0.2949], 0.3978$, & $([0.2289,0.3422], 0.4485$, \\
& $[0.5333,0.6336])$ & $[0.6952,0.7976])$ & $[0.4995,0.6006])$ & $[0.5522,0.6547])$ \\
\hline
\end{tabular}

TABLE 6: Score values obtained by the TIT2FFWA operator and the rankings of alternatives.

\begin{tabular}{lccccc}
\hline & $A_{1}$ & $A_{2}$ & $A_{3}$ & $A_{4}$ & Ranking \\
\hline$\lambda \rightarrow 1$ & 1.2090 & 1.2746 & 0.9107 & 0.8817 & $A_{2}>A_{1}>A_{3}>A_{4}$ \\
$\lambda=2$ & 1.2027 & 1.2719 & 0.9022 & 0.8721 & $A_{2}>A_{1}>A_{3}>A_{4}$ \\
$\lambda=5$ & 1.2008 & 1.2685 & 0.8943 & 0.8687 & $A_{2}>A_{1}>A_{3}>A_{4}$ \\
$\lambda=10$ & 1.2003 & 1.2663 & 0.8894 & 0.8645 & $A_{2}>A_{1}>A_{3}>A_{4}$ \\
$\lambda=50$ & 1.1971 & 1.2652 & 0.8872 & 0.8624 & $A_{2}>A_{1}>A_{3}>A_{4}$ \\
$\lambda \rightarrow+\infty$ & 1.1184 & 1.1924 & 0.7271 & 0.7020 & $A_{2}>A_{1}>A_{3}>A_{4}$ \\
\hline
\end{tabular}


TABLE 7: Score values obtained by the TIT2FFWG operator and the rankings of alternatives.

\begin{tabular}{lccccc}
\hline & $A_{1}$ & $A_{2}$ & $A_{3}$ & $A_{4}$ & Ranking \\
\hline$\lambda \rightarrow 1$ & 1.1082 & 1.1735 & 0.6803 & 0.6662 & $A_{2}>A_{1}>A_{3}>A_{4}$ \\
$\lambda=2$ & 1.1113 & 1.1774 & 0.6866 & 0.6710 & $A_{2}>A_{1}>A_{3}>A_{4}$ \\
$\lambda=5$ & 1.1133 & 1.1836 & 0.6942 & 0.6767 & $A_{2}>A_{1}>A_{3}>A_{4}$ \\
$\lambda=10$ & 1.1144 & 1.1841 & 0.6989 & 0.6805 & $A_{2}>A_{1}>A_{3}>A_{4}$ \\
$\lambda=50$ & 1.1151 & 1.1843 & 0.7023 & 0.7004 & $A_{2}>A_{1}>A_{3}>A_{4}$ \\
$\lambda \rightarrow+\infty$ & 1.1184 & 1.1924 & 0.7271 & 0.7020 & $A_{2}>A_{1}>A_{3}>A_{4}$ \\
\hline
\end{tabular}

Based on the TIT2FFWG operator, in order to select the most desirable supplier, we can also develop a method for handling MAGDM problems with triangular interval type-2 fuzzy information, which can be described as follows.

Step $1^{\prime}$. It is the same as Step 1.

Step $2^{\prime}$. Utilize the TIT2FFWG operator to aggregate all the individual triangular interval type-2 fuzzy decision matrix $\widetilde{R}^{(k)}=\left(\widetilde{r}_{i j}^{(k)}\right)_{4 \times 4}$ into the collective triangular interval type- 2 fuzzy decision matrix $\widetilde{R}=\left(\widetilde{r}_{i j}\right)_{4 \times 4}$, and without the loss of generality, we let $\lambda=2$; the result is shown in Table 5 .

Step $3^{\prime}$. Utilize the TIT2FFWG operator to aggregate all the preference values $\widetilde{r}_{i j}(j=1,2,3,4)$ in the ith line of $\widetilde{R}$, and then we can get the overall preference values $\widetilde{r}_{i}(i=1,2,3,4)$ as follows:

$$
\begin{aligned}
\widetilde{r}_{1} & =([0.4655,0.5663], 0.6669,[0.7674,0.8677]), \\
\widetilde{r}_{2} & =([0.4918,0.5938], 0.6951,[0.7961,0.8969]), \\
\widetilde{r}_{3} & =([0.2531,0.3645], 0.4704,[0.5741,0.6766]), \\
\widetilde{r}_{4} & =([0.2488,0.3571], 0.4616,[0.5644,0.6663]) .
\end{aligned}
$$

Step $4^{\prime}$. Calculate the score values $S\left(\widetilde{r}_{i}\right)(i=1,2,3,4)$ of the overall preference values $\widetilde{r}_{i}(i=1,2,3,4)$, respectively:

$$
\begin{array}{ll}
S\left(\widetilde{r}_{1}\right)=1.1113, & S\left(\widetilde{r}_{2}\right)=1.1774, \\
S\left(\widetilde{r}_{3}\right)=0.6866, & S\left(\widetilde{r}_{4}\right)=0.6710 .
\end{array}
$$

Step $5^{\prime}$. Rank all the alternatives $A_{i}(i=1,2,3,4)$ and select the best one(s) in accordance with $S\left(\widetilde{r}_{i}\right)$.

Since

$$
S\left(\widetilde{r}_{2}\right)>S\left(\widetilde{r}_{1}\right)>S\left(\widetilde{r}_{3}\right)>S\left(\widetilde{r}_{4}\right)
$$

therefore, we have

$$
A_{2}>A_{1}>A_{3}>A_{4},
$$

where the symbol " $>$ " means "superior to." Thus, the best supplier is $A_{2}$.

It is clear to see that they are the same ranking results for two methods based on TIT2FFWA operator and the TIT2FFWG operator. In order to reflect the influence with the
TABLE 8: Comparisons with type-2 OWA and type-2 FWA.

\begin{tabular}{lccc}
\hline $\begin{array}{l}\text { Aggregation } \\
\text { operator }\end{array}$ & $\begin{array}{c}\text { Computational } \\
\text { complexity }\end{array}$ & Flexible & Order of alternatives \\
\hline $\begin{array}{l}\text { Type-2 OWA } \\
\text { operator }\end{array}$ & High & None & $A_{2}>A_{1}>A_{3}>A_{4}$ \\
$\begin{array}{l}\text { Type-2 FWA } \\
\text { operator }\end{array}$ & Very high & None & $A_{2}>A_{1}>A_{3}>A_{4}$ \\
$\begin{array}{l}\text { The proposed } \\
\text { operators }\end{array}$ & Low & High & $A_{2}>A_{1}>A_{3}>A_{4}$ \\
\hline
\end{tabular}

different value of parameter $\lambda$, we use different parameter $\lambda$ in our proposed methods to rank the alternatives. The ranking results are shown in Tables 6 and 7.

As we can see from Tables 6 and 7, the aggregation score values based on TIT2FFWA and TIT2FFWG operators with different parameter $\gamma$ are different, but the ranking orders of the alternatives are the same in this example. The decision makers can choose the appropriate value in accordance with their preferences. By further analyzing Tables 6 and 7, we can easily see that the score values obtained by the TIT2FFWA operator became smaller as the parameter $\lambda$ increases for the same aggregation elements, but the TIT2FFWG operator became greater as the parameter $\lambda$ increases for the same aggregation elements. Furthermore, the values obtained by the TIT2FFWA operator are always larger than the ones obtained by the TIT2FFWG operator for the same value of the parameter $\lambda$ and the same aggregation elements.

In order to verify the validity of our methods, we make a comparison between our proposed operators with the type-2 OWA operator [46] and the type-2 fuzzy weighted averaging operator [62] for solving multiple attribute group decision making with interval type-2 fuzzy information. Due to the space limit, we give the results directly. The ranking results are shown in Table 8.

From Table 8, we can see that our methods can obtain the same ranking results with the type-2 OWA operator proposed by Zhou et al. [46] and type-2 FWA operator proposed by Liu and Wang [62] in this example. This fact verifies that the TIT2FFWA and TIT2FFWG operators we proposed are reasonable and valid for interval type-2 fuzzy decision-making problems. Compared with the type-2 OWA operator and the type-2 FWA operator, the main advantage of TIT2FFWA and TIT2FFWG operators we proposed is that it can reduce the cost of computational complexity and enhance the reliability of the aggregation result under fuzzy environment. Furthermore, our proposed methods include 
a parameter, which can adjust the aggregate value based on the real decision needs and reflect the decision maker's risk preferences. Therefore, the benefit is that the proposed operators are more general and more flexible.

According to the comparisons and analysis above, our proposed aggregation operators are better than the other two methods.

\section{Conclusions}

This paper puts forward a new method to solve MAGDM with triangle interval type-2 fuzzy information. We have defined Frank operation laws of interval type-2 fuzzy set, and by using Frank triangular norms, some new aggregation operators, including the TIT2FFWA and TIT2FFWG operators, are developed. The properties of these operators have been discussed in detail. Moreover, we have applied the TIT2FFWA and TIT2FFWG operators for handling MAGDM problems under the triangle interval type- 2 fuzzy environment. Finally, an illustrative example about supplier selection has been provided to demonstrate how to apply the proposed procedure. In further research, we will continue our working and extend the developed aggregation operators to the fields of data mining [63], technology evaluation [64], computing with words [65], and cluster analysis [66].

\section{Conflict of Interests}

The authors declare that there is no conflict of interests regarding the publication of this paper.

\section{Acknowledgments}

The work is supported by the National Natural Science Foundation of China (NSFC) under Projects 71171048 and 71371049, Ph.D. Program Foundation of Chinese Ministry of Education 20120092110038, and the Scientific Research and Innovation Project for College Graduates of Jiangsu Province CXZZ13_0138.

\section{References}

[1] D. Li and J. Yang, "Fuzzy linear programming technique for multiattribute group decision making in fuzzy environments," Information Sciences, vol. 158, pp. 263-275, 2004.

[2] Y. J. Wang and H. S. Lee, "Generalizing TOPSIS for fuzzy multiple-criteria group decision-making," Computers and Mathematics with Applications, vol. 53, no. 11, pp. 1762-1772, 2007.

[3] Z. Xu and J. Chen, "An interactive method for fuzzy multiple attribute group decision making," Information Sciences, vol. 177, no. 1, pp. 248-263, 2007.

[4] C. J. Lin and W. W. Wu, "A causal analytical method for group decision-making under fuzzy environment," Expert Systems with Applications, vol. 34, no. 1, pp. 205-213, 2008.

[5] D. Guha and D. Chakraborty, "Fuzzy multi attribute group decision making method to achieve consensus under the consideration of degrees of confidence of experts' opinions,"
Computers and Industrial Engineering, vol. 60, no. 4, pp. 493504, 2011.

[6] A. Hatami-Marbini and M. Tavana, "An extension of the Electre I method for group decision-making under a fuzzy environment," Omega, vol. 39, no. 4, pp. 373-386, 2011.

[7] S. M. Mousavi, F. Jolai, and R. Tavakkoli-Moghaddam, "A Fuzzy stochastic multi-attribute group decision-making approach for selection problems," Group Decision and Negotiation, vol. 22, no. 2, pp. 207-233, 2013.

[8] X. Zhao, R. Lin, and G. Wei, "Fuzzy prioritized operators and their application to multiple attribute group decision making," Applied Mathematical Modelling, vol. 37, no. 7, pp. 4759-4770, 2013.

[9] L. A. Zadeh, "The concept of a linguistic variable and its application to approximate reasoning-I," Information Sciences, vol. 8, no. 3, pp. 199-249, 1975.

[10] J. M. Mendel, "Computing with words and its relationships with fuzzistics," Information Sciences, vol. 177, no. 4, pp. 988-1006, 2007.

[11] I. B. Turksen, "Type 2 representation and reasoning for CWW," Fuzzy Sets and Systems, vol. 127, no. 1, pp. 17-36, 2002.

[12] N. N. Karnik and J. M. Mendel, "Centroid of a type-2 fuzzy set," Information Sciences, vol. 132, no. 1-4, pp. 195-220, 2001.

[13] N. N. Karnik and J. M. Mendel, "Operations on type-2 fuzzy sets," Fuzzy Sets and Systems, vol. 122, no. 2, pp. 327-348, 2001.

[14] F. Liu, "An efficient centroid type-reduction strategy for general type-2 fuzzy logic system," Information Sciences, vol. 178, no. 9, pp. 2224-2236, 2008.

[15] J. M. Mendel, “Type-2 fuzzy sets and systems: an overview," IEEE Computational Intelligence Magazine, vol. 2, no. 1, pp. 20 29, 2007.

[16] D. Wu and J. M. Mendel, "Enhanced Karnik-Mendel algorithms," IEEE Transactions on Fuzzy Systems, vol. 17, no. 4, pp. 923-934, 2009.

[17] D. Y. Zhai and J. M. Mendel, "Enhanced centroid-flow algorithm for computing the centroid of general type-2 fuzzy sets," IEEE Transactions on Fuzzy Systems, vol. 20, no. 5, pp. 939-956, 2012.

[18] J. M. Mendel, F. Liu, and D. Zhai, “ $\alpha$-plane representation for type-2 fuzzy sets: theory and applications," IEEE Transactions on Fuzzy Systems, vol. 17, no. 5, pp. 1189-1207, 2009.

[19] C. Hwang, M. Yang, and W. Hung, "On similarity, inclusion measure and entropy between type-2 fuzzy sets," International Journal of Uncertainty, Fuzziness and Knowledge-Based Systems, vol. 20, no. 3, pp. 433-449, 2012.

[20] H. B. Mitchell, "Pattern recognition using type-II fuzzy sets," Information Sciences, vol. 170, no. 2-4, pp. 409-418, 2005.

[21] H. R. Tizhoosh, "Image thresholding using type II fuzzy sets," Pattern Recognition, vol. 38, no. 12, pp. 2363-2372, 2005.

[22] J. Aisbett, J. T. Rickard, and D. Morgenthaler, "Multivariate modeling and type-2 fuzzy sets," Fuzzy Sets and Systems, vol. 163, pp. 78-95, 2011.

[23] J. M. Mendel, R. I. John, and F. Liu, "Interval type-2 fuzzy logic systems made simple," IEEE Transactions on Fuzzy Systems, vol. 14, no. 6, pp. 808-821, 2006.

[24] H. Zhou and H. Ying, "A method for deriving the analytical structure of a broad class of typical interval type-2 mamdani fuzzy controllers," IEEE Transactions on Fuzzy Systems, vol. 21, no. 3, pp. 447-458, 2013. 
[25] A. Abbadi, L. Nezli, and D. Boukhetala, "A nonlinear voltage controller based on interval type 2 fuzzy logic control system for multimachine power systems," International Journal of Electrical Power and Energy Systems, vol. 45, no. 1, pp. 456-467, 2013.

[26] Y. Maldonado, O. Castillo, and P. Melin, "Particle swarm optimization of interval type-2 fuzzy systems for FPGA applications," Applied Soft Computing Journal, vol. 13, no. 1, pp. 496$508,2013$.

[27] S. T. Wang, F. L. Chung, Y. Y. Li, D. W. Hu, and X. S. Wu, "A new gaussian noise filter based on interval type-2 fuzzy logic systems," Soft Computing, vol. 9, no. 5, pp. 398-406, 2005.

[28] B. Choi and F. Chung-Hoon Rhee, "Interval type-2 fuzzy membership function generation methods for pattern recognition," Information Sciences, vol. 179, no. 13, pp. 2102-2122, 2009.

[29] D. Akay, O. Kulak, and B. Henson, "Conceptual design evaluation using interval type-2 fuzzy information axiom," Computers in Industry, vol. 62, no. 2, pp. 138-146, 2011.

[30] J. Hwang, H. Kwak, and G. Park, "Adaptive interval type-2 fuzzy sliding mode control for unknown chaotic system," Nonlinear Dynamics, vol. 63, no. 3, pp. 491-502, 2011.

[31] S. Chen and L. Lee, "Fuzzy multiple criteria hierarchical group decision-making based on interval type-2 fuzzy sets," IEEE Transactions on Systems, Man, and Cybernetics A: Systems and Humans, vol. 40, no. 5, pp. 1120-1128, 2010.

[32] S. M. Chen and L. W. Lee, "Fuzzy multiple attributes group decision-making based on the interval type-2 TOPSIS method," Expert Systems with Applications, vol. 37, no. 4, pp. 2790-2798, 2010.

[33] S. M. Chen, M. W. Yang, L. W. Lee, and S. W. Yang, "Fuzzy multiple attributes group decision-making based on ranking interval type-2 fuzzy sets," Expert Systems with Applications, vol. 39, no. 5, pp. 5295-5308, 2012.

[34] J. H. Hu, Y. Zhang, X. H. Chen, and Y. M. Liu, "Multi-criteria decision making method based on possibility degree of interval type-2 fuzzy number," Knowledge-Based Systems, vol. 43, pp. 2129, 2013.

[35] W. Wang, X. Liu, and Y. Qin, "Multi-attribute group decision making models under interval type-2 fuzzy environment," Knowledge-Based Systems, vol. 30, pp. 121-128, 2012.

[36] T. Chen, "A signed-distance-based approach to importance assessment and multi-criteria group decision analysis based on interval type-2 fuzzy set," Knowledge and Information Systems, vol. 35, no. 1, pp. 193-231, 2013.

[37] T. Chen, C. Chang, and J. R. Lu, “The extended QUALIFLEX method for multiple criteria decision analysis based on interval type-2 fuzzy sets and applications to medical decision making," European Journal of Operational Research, vol. 226, no. 3, pp. 615-625, 2013.

[38] T. Baležentis and S. Zeng, "Group multi-criteria decision making based upon interval-valued fuzzy numbers: an extension of the MULTIMOORA method," Expert Systems with Applications, vol. 40, no. 2, pp. 543-550, 2013.

[39] K. P. Chiao, "Multiple criteria group decision making with triangular interval type-2 fuzzy sets," in Proceedings of the IEEE International Conference on Fuzzy Systems (FUZZ '11), pp. 25752582, Taipei, Taiwan, June 2011.

[40] S.-C. Ngan, "A type-2 linguistic set theory and its application to multi-criteria decision making," Computers \& Industrial Engineering, vol. 64, no. 2, pp. 721-730, 2013.

[41] T. Chen, "A linear assignment method for multiple-criteria decision analysis with interval type-2 fuzzy sets," Applied Soft Computing Journal, vol. 13, no. 5, pp. 2735-2748, 2013.
[42] Z. M. Zhang and S. H. Zhang, "A novel approach to multi attribute group decision making based on trapezoidal interval type-2 fuzzy soft sets," Applied Mathematical Modelling, vol. 37, no. 7, pp. 4948-4971, 2013.

[43] D. Wu and J. M. Mendel, "Aggregation using the linguistic weighted average and interval type-2 fuzzy sets," IEEE Transactions on Fuzzy Systems, vol. 15, no. 6, pp. 1145-1161, 2007.

[44] X. Liu, J. M. Mendel, and D. Wu, "Analytical solution methods for the fuzzy weighted average," Information Sciences, vol. 187, pp. 151-170, 2012.

[45] S. M. Zhou, F. Chiclana, R. I. John, and J. M. Garibaldi, “Type-2 OWA operators: aggregating type-2 fuzzy sets in soft decision making," in Proceedings of the IEEE International Conference on Fuzzy Systems (FUZZ '08), vol. 1-5, pp. 625-630, June 2008.

[46] S. M. Zhou, R. I. John, F. Chiclana, and J. M. Garibaldi, "On aggregating uncertain information by type-2 OWA operators for soft decision making," International Journal of Intelligent Systems, vol. 25, no. 6, pp. 540-558, 2010.

[47] P. Liu, "A weighted aggregation operators multi-attribute group decision-making method based on interval-valued trapezoidal fuzzy numbers," Expert Systems with Applications, vol. 38, no. 1, pp. 1053-1060, 2011.

[48] P. D. Liu and F. Jin, "A multi-attribute group decision-making method based on weighted geometric aggregation operators of interval-valued trapezoidal fuzzy numbers," Applied Mathematical Modelling, vol. 36, no. 6, pp. 2498-2509, 2012.

[49] G. Deschrijver, C. Cornelis, E. E. Kerre, and G. Deschrijver, "On the representation of intuitionistic fuzzy t-norms and tconorms," IEEE Transactions on Fuzzy Systems, vol. 12, no. 1, pp. 45-61, 2004.

[50] G. Deschrijver and E. E. Kerre, "Uninorms in $\mathrm{L}^{*}$-fuzzy set theory," Fuzzy Sets and Systems, vol. 148, no. 2, pp. 243-262, 2004.

[51] G. Deschrijver and E. E. Kerre, "Implicators based on binary aggregation operators in interval-valued fuzzy set theory," Fuzzy Sets and Systems, vol. 153, no. 2, pp. 229-248, 2005.

[52] M. Xia, Z. Xu, and B. Zhu, "Some issues on intuitionistic fuzzy aggregation operators based on Archimedean t-conorm and tnorm," Knowledge-Based Systems, vol. 31, pp. 78-88, 2012.

[53] W. Z. Wang and X. W. Liu, "Intuitionistic fuzzy information aggregation using einstein operations," IEEE Transactions on Fuzzy Systems, vol. 20, no. 5, pp. 923-938, 2012.

[54] P. D. Liu, "Some Hamacher aggregation operators based on the interval-valued intuitionistic fuzzy numbers and their application to group decision making," IEEE Transactions on Fuzzy Systems, vol. 22, no. 1, pp. 83-97, 2014.

[55] M. J. Frank, "On the simultaneous associativity of $F(x, y)$ and $x+y-F(x, y)$," Aequationes Mathematicae, vol. 19, no. 1, pp. 194-226, 1979.

[56] G. Beliakov, A. Pradera, and T. Calvo, Aggregation Functions: A Guide for Practitioners, Springer, Heidelberg, Germany, 2007.

[57] T. Calvo, B. De Baets, and J. Fodor, "The functional equations of Frank and Alsina for uninorms and nullnorms," Fuzzy Sets and Systems, vol. 120, no. 3, pp. 385-394, 2001.

[58] R. R. Yager, "On some new classes of implication operators and their role in approximate reasoning," Information Sciences, vol. 167, no. 1-4, pp. 193-216, 2004.

[59] J. Casanovas and J. Torrens, "An axiomatic approach to fuzzy cardinalities of finite fuzzy sets," Fuzzy Sets and Systems, vol. 133, no. 2, pp. 193-209, 2003. 
[60] P. Sarkoci, "Domination in the families of Frank and Hamacher t-norms," Kybernetika, vol. 41, no. 3, pp. 349-360, 2005.

[61] W. Wang and H. He, "Research on flexible probability logic operator based on Frank T/S norms," Acta Electronica Sinica, vol. 37, no. 5, pp. 1141-1145, 2009.

[62] X. Liu and Y. Wang, "An analytical solution method for the generalized fuzzy weighted average problem," International Journal of Uncertainty, Fuzziness and Knowledge-Based Systems, vol. 21, no. 3, pp. 455-480, 2013.

[63] C. Qiu, J. Xiao, L. Yu, L. Han, and M. N. Iqbal, "A modified interval type-2 fuzzy C-means algorithm with application in MR image segmentation," Pattern Recognition Letters, vol. 34, no. 12, pp. 1329-1338, 2013.

[64] T. Dereli and K. Altun, "Technology evaluation through the use of interval type-2 fuzzy sets and systems," Computers and Industrial Engineering, vol. 65, no. 4, pp. 624-633, 2013.

[65] D. Wu and J. M. Mendel, "Computing with words for hierarchical decision making applied to evaluating a weapon system," IEEE Transactions on Fuzzy Systems, vol. 18, no. 3, pp. 441-460, 2010.

[66] H. Le Capitaine and C. Frélicot, "A cluster-validity index combining an overlap measure and a separation measure based on fuzzy-aggregation operators," IEEE Transactions on Fuzzy Systems, vol. 19, no. 3, pp. 580-588, 2011. 


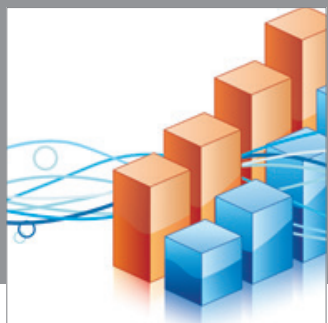

Advances in

Operations Research

mansans

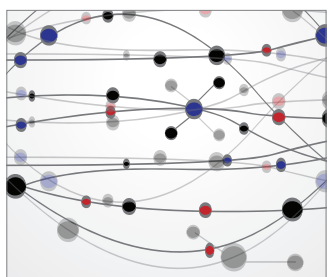

The Scientific World Journal
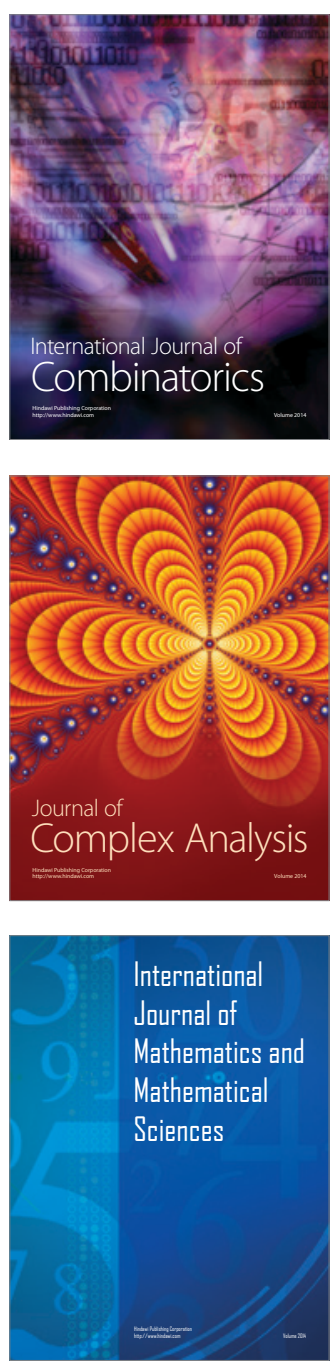
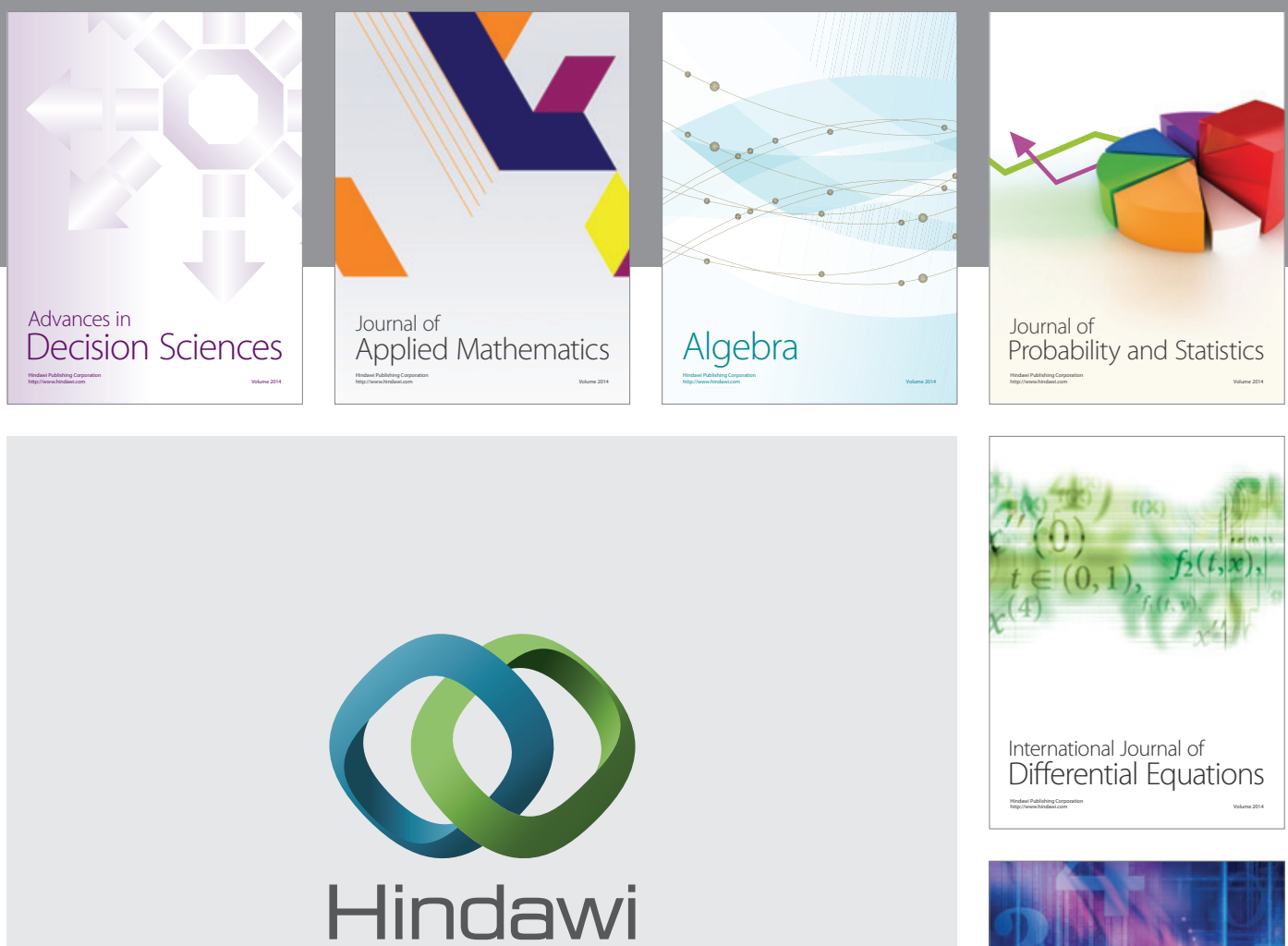

Submit your manuscripts at http://www.hindawi.com
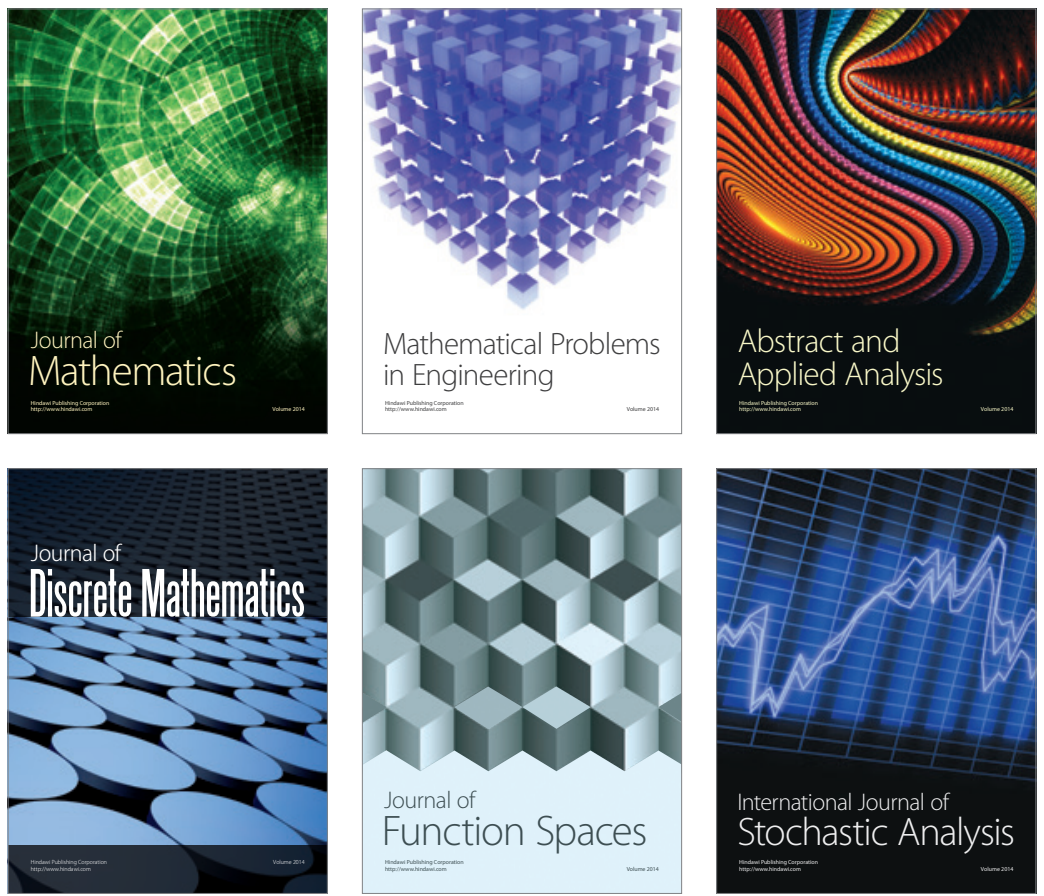

Journal of

Function Spaces

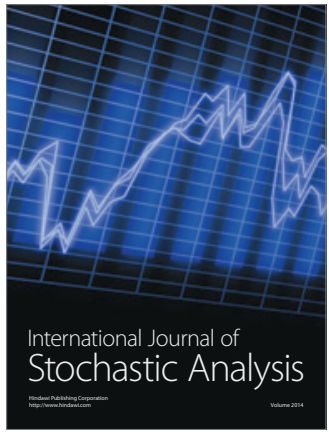

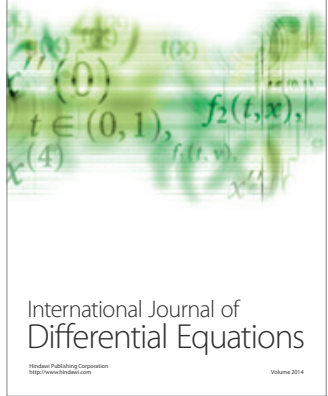
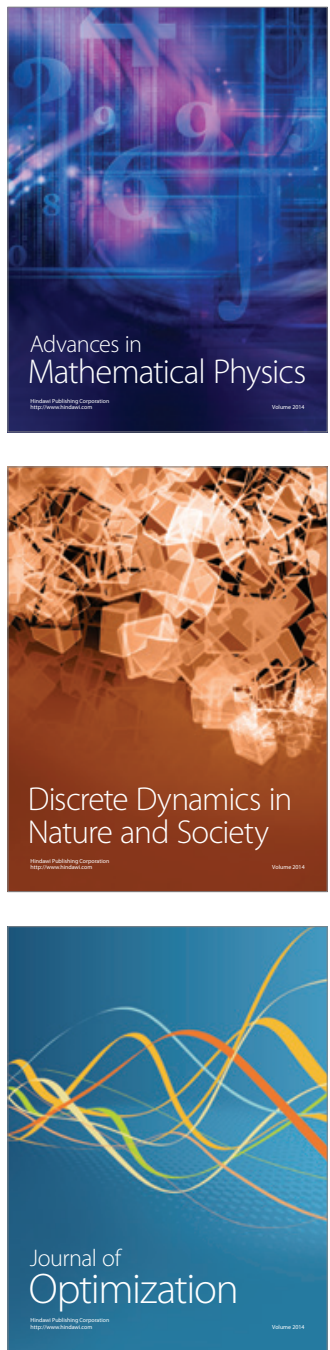